TRANSACTIONS OF THE

AMERICAN MATHEMATICAL SOCIETY

Volume 362, Number 9, September 2010, Pages 4617-4653

S 0002-9947(10)05003-8

Article electronically published on April 9, 2010

\title{
MULTIDIMENSIONAL SOFIC SHIFTS WITHOUT SEPARATION AND THEIR FACTORS
}

\author{
MIKE BOYLE, RONNIE PAVLOV, AND MICHAEL SCHRAUDNER
}

\begin{abstract}
For $d \geq 2$ we exhibit mixing $\mathbb{Z}^{d}$ shifts of finite type and sofic shifts with large entropy but poorly separated subsystems (in the sofic examples, the only minimal subsystem is a single point). These examples consequently have very constrained factors; in particular, no non-trivial full shift is a factor. We also provide examples to distinguish certain mixing conditions and develop the natural class of "block gluing" shifts. In particular, we show that block gluing shifts factor onto all full shifts of strictly smaller entropy.
\end{abstract}

\section{INTRODUCTION}

The $\mathbb{Z}^{d}$ shifts of finite type (SFTs) and sofic shifts $(d \geq 2)$ are a drastically more problematic and diverse class of systems than their relatives in only one dimension 24, 26, 28. The most fundamental theorems in the $\mathbb{Z}$ case do not carry over to the $\mathbb{Z}^{d}$ setting for $d \geq 2$, and nearly all structural results $4,5,9,12,14,16,17,18,24,27$. hold only for certain subclasses of $\mathbb{Z}^{d} \mathrm{SFTs}$ or $\mathbb{Z}^{d}$ sofic shifts (for a rare exception, see 8 ). Non-trivial mixing $\mathbb{Z}^{d}$ SFTs are qualitatively homogeneous in the case $d=1$, but quite heterogeneous for $d \geq 2$.

As part of the exploration of this landscape of possibilities, we consider subsystems and factors. Every mixing $\mathbb{Z}$ SFT or sofic shift of positive entropy contains a vast collection of pairwise disjoint subsystems [4.5). For $d \geq 2$, Desai has shown that a general $\mathbb{Z}^{d}$ sofic shift $X$ of positive entropy still contains many subsystems: it contains subshifts achieving all entropies in the interval $[0, h(X)]$. What we emphasize in this paper is that, in contrast to the $\mathbb{Z}$ case, these subsystems may be very poorly separated. For example, we exhibit mixing $\mathbb{Z}^{d}$ sofic shifts of arbitrarily large entropy whose only minimal subsystem is a fixed point (so no pair of subsystems can be disjoint). Similarly, for $d \geq 2$ we exhibit $\mathbb{Z}^{d}$ SFTs of arbitrarily large entropy, all of whose minimal subsystems are contained in a subSFT of zero entropy.

Under a factor map, the preimages of disjoint subsystems are disjoint. Consequently systems with poorly separated subsystems cannot factor onto systems with a rich collection of pairwise disjoint subsystems. In particular, our examples cannot

Received by the editors June 25, 2008.

2010 Mathematics Subject Classification. Primary 37B50; Secondary 37B10, 37A35, 37A15.

Key words and phrases. $\mathbb{Z}^{d}$, shift of finite type, sofic, minimal subshift, factor, subsystem, mixing, entropy, multidimensional.

The first author was partly supported by Dassault Chair, Nucleus Millennium P04-069-F, NSF Grant 0400493, and Basal-CMM grant.

The third author was supported by FONDECYT project 3080008 .

(C)2010 American Mathematical Society Reverts to public domain 28 years from publication 
factor onto any non-trivial block gluing SFT, and in particular cannot factor onto a non-trivial full shift. This provides a negative answer to the question of Johnson and Madden studied in [14, 9, 5, 11] and is another stark contrast to the case $d=1$ (4.6). The sofic examples cannot factor onto or contain a non-trivial SFT, again in stark contrast to the $\mathbb{Z}$ case (4.6). In some cases, our examples can be arranged to have topologically completely positive entropy, but at the same time to have no factor admitting an invariant measure such that as a measurable system it is of completely positive entropy. The proof (6.1) of the latter property appeals to a $\mathbb{Z}^{d}$ disjointness theorem of Glasner, Thouvenot and Weiss [10].

The paper is organized as follows. In Section 2, we provide symbolic dynamics background. In Section 3, we define a natural notion of "block gluing" (mixing on blocks given a uniform separation) and show for $d \geq 2$ that any block gluing $\mathbb{Z}^{d}$ shift $X$ has many factors, including full shifts of any smaller entropy and a set of SFTs with entropies dense in $[0, h(X)]$. This generalizes the corresponding result of Johnson-Madden-Desai for corner gluing shifts [14, 9]. In Section 4, we state our main results on systems with restricted subsystems and factors, and contrast them with the $\mathbb{Z}$ case. The proofs are carried out over Sections 5] 10 as described in Section 4. The constructions are different for the cases $d=2$ and $d \geq 3$, and rely heavily on two papers of Mike Hochman [11, 12.

In the appendices, we establish some properties of block gluing shifts, discuss a range of mixing conditions on $\mathbb{Z}^{d}$ shifts $(d \geq 2)$, and provide some examples. We show the uniform filling property is not implied by corner gluing, block gluing does not imply corner gluing, and a block gluing (or even corner gluing) $\mathbb{Z}^{d}$ SFT need not be entropy minimal.

\section{BACKGROUND}

We assume a basic familiarity with symbolic dynamics; nevertheless we recall some notation in this section. Formal definitions of some relevant mixing notions so far introduced to the study of $\mathbb{Z}^{d}$ shifts by other authors can be found in Appendix A. where we also discuss their mutual relations.

A $d$-dimensional full shift $(d \in \mathbb{N})$ on some finite alphabet $\mathcal{A}$ consists of the set $\mathcal{A}^{\mathbb{Z}^{d}}$ together with the $\mathbb{Z}^{d}$ shift action $\sigma^{\vec{\imath}}: \mathcal{A}^{\mathbb{Z}^{d}} \rightarrow \mathcal{A}^{\mathbb{Z}^{d}}\left(\vec{\imath} \in \mathbb{Z}^{d}\right)$ defined at each coordinate $\vec{\jmath} \in \mathbb{Z}^{d}$ as $\left(\sigma^{\vec{\imath}}(x)\right)_{\vec{\jmath}}:=x_{\vec{\imath}+\vec{\jmath}}$.

Closed shift-invariant subsets of some $d$-dimensional full shift are called $\mathbb{Z}^{d}$ (sub)shifts. A subsystem of some $\mathbb{Z}^{d}$ shift $X$ is a closed shift-invariant subset of $X$, together with the restriction of the $\mathbb{Z}^{d}$ shift action to this set.

The language of a $\mathbb{Z}^{d}$ shift $X$ is the set of finite patterns which appear as subwords of elements of $X$. It is denoted by $\mathcal{L}(X)$. The subset of patterns in the language of $X$ with a certain (finite) shape $F \subsetneq \mathbb{Z}^{d}$ we denote by $\mathcal{L}_{F}(X):=\left\{\left.x\right|_{F} \mid x \in X\right\} \subsetneq \mathcal{L}(X)$.

For any $\mathbb{Z}^{d}$ shift $X$, pattern $P \in \mathcal{L}_{F}(X)$, and point $x \in X$, the frequency of $P$ in $x$ (if it exists) is given as

$$
\lim _{n \rightarrow \infty} \frac{\left|\left\{\vec{\imath} \in F_{n}|x|_{F+\vec{\imath}}=P\right\}\right|}{\left|F_{n}\right|}
$$

where $F_{n}:=\left\{\vec{\imath} \in \mathbb{Z}^{d} \mid\|\vec{\imath}\|_{\infty} \leq n\right\}$.

A $d$-dimensional shift of finite type $\left(\mathbb{Z}^{d} \mathrm{SFT}\right)$ is a $\mathbb{Z}^{d}$ shift $X \subseteq \mathcal{A}^{\mathbb{Z}^{d}}$ defined using a finite set $\mathcal{P} \subseteq \mathcal{A}^{F}$ of patterns on a finite non-empty shape $F \subsetneq \mathbb{Z}^{d}$, such that 
$X=\left\{x \in \mathcal{A}^{\mathbb{Z}^{d}}\left|\forall \vec{\imath} \in \mathbb{Z}^{d}: x\right|_{\vec{\imath}+F} \in \mathcal{P}\right\}$. If the diameter of $F$ with respect to the maximum-norm $\|\cdot\|_{\infty}$ on $\mathbb{Z}^{d}$ is $m \in \mathbb{N}$, then $X$ has order $m$.

Recall that the topological entropy of a $\mathbb{Z}^{d}$ shift $X$ is by definition

$$
h(X):=\lim _{n \rightarrow \infty} \frac{\log \left|\mathcal{L}_{F_{n}}(X)\right|}{\left|F_{n}\right|},
$$

where $F_{n}:=\left\{\vec{\imath} \in \mathbb{Z}^{d} \mid\|\vec{\imath}\|_{\infty} \leq n\right\}$. (Note that by [1] this limit $h(X)$ is the same for any sequence of finite convex shapes $\left(F_{n} \subsetneq \mathbb{Z}^{d}\right)_{n \in \mathbb{N}}$, such that the inradii of $F_{n}$ diverge to infinity with $n$.) The measure-theoretic entropy $h_{\mu}(X)$ of $X$ with respect to a shift-invariant Borel probability $\mu \in \mathcal{M}(X)$ is generalized analogously from the $\mathbb{Z}$ case.

Unless qualified, by a dynamical system we mean in this paper a homeomorphism of a compact metric space. We call a point $x$ of a dynamical system $(\Xi, \xi)$ transitive if its $\xi$-orbit is dense in $\Xi$. A dynamical system $(\Xi, \xi)$ is called minimal if all points are transitive, or in other words if all $\xi$-orbits are dense in $\Xi$, meaning that $\Xi$ itself is the only non-empty $\xi$-invariant closed subset of $\Xi$. A system $(\Xi, \xi)$ is called trivial if it consists of only one point.

A factor map between two $\mathbb{Z}^{d}$ shifts means a surjective map intertwining the shift actions, and the image of a subshift $X$ under such a map is referred to as a factor of $X$. A factor map is called topological if it is in addition continuous, and it is called measurable if it is a measure preserving Borel map. In this paper, unless indicated otherwise, factor maps are in the topological category. It is well known that factor maps never increase entropy.

The class of $\mathbb{Z}^{d}$ sofic shifts constitutes the factors of $\mathbb{Z}^{d}$ SFTs. Obviously it is closed under factor maps, i.e. factors of sofic shifts are again sofic.

By a measurable dynamical system we mean in this paper an invertible measure preserving transformation of a Borel probability space. We say a measurable dynamical system has (measurably) completely positive entropy (c.p.e.) if every non-trivial measurable factor has positive entropy. Likewise we say a topological dynamical system has (topologically) completely positive entropy (top.c.p.e.) if every non-trivial topological factor has positive entropy.

\section{Factors of BLOCK GLUing $\mathbb{Z}^{d}$ Shifts $(d \geq 1)$}

In this section we introduce the uniform mixing property "block gluing" for $\mathbb{Z}^{d}$ shifts, and we prove that block gluing $\mathbb{Z}^{d}$ shifts factor onto full shifts of smaller entropy (and more generally onto any lower entropy $\mathbb{Z}^{d}$ SFT containing a safe symbol) as well as onto a family of strongly irreducible $\mathbb{Z}^{d}$ SFTs, realizing a dense set of values in the possible range of entropies. We discuss block gluing shifts in detail in Appendix B.

In the sequel, $\delta_{\infty}$ will represent the maximum-metric on $\mathbb{Z}^{d}$; i.e. $\forall \vec{v}, \vec{w} \in \mathbb{Z}^{d}$ we define $\delta_{\infty}(\vec{v}, \vec{w}):=\|\vec{v}-\vec{w}\|_{\infty}=\max _{1 \leq k \leq d}\left|v_{k}-w_{k}\right| . \quad \delta_{\infty}$ can be extended to a non-negative symmetric function on non-empty subsets $V, W \subseteq \mathbb{Z}^{d}$ in the usual way: $\delta_{\infty}(V, W):=\min _{\vec{v} \in V, \vec{w} \in W} \delta_{\infty}(\vec{v}, \vec{w})$.

Let $\vec{v}, \vec{w} \in \mathbb{Z}^{d}$ such that for all $1 \leq k \leq d$ the components satisfy $\vec{v}_{k} \leq \vec{w}_{k}$. We will denote this with $\vec{v} \preceq \vec{w}$. For every pair $\vec{v} \preceq \vec{w}$ the non-empty subset $B:=\mathbb{Z}^{d} \cap \prod_{k=1}^{d}\left[\vec{v}_{k}, \vec{w}_{k}\right]=\left\{\vec{\imath} \in \mathbb{Z}^{d} \mid \vec{v} \preceq \vec{\imath} \preceq \vec{w}\right\}$ constitutes a solid block for which we use the notation $B=[\vec{v}, \vec{w}]$. With $\overrightarrow{\mathbb{1}}$ we refer to the element in $\mathbb{Z}^{d}$ in which all 
components equal 1, i.e. $\overrightarrow{\mathbb{1}}:=(1,1, \ldots, 1) \in \mathbb{Z}^{d}$ and by $\mathbb{N}_{0}$ to the set of non-negative integers.

Definition 3.1. A $\mathbb{Z}^{d}$ shift $X$ is called block gluing at gap $g \in \mathbb{N}_{0}$ if for any two (disjoint) solid blocks $B_{1}=\left[\vec{v}^{(1)}, \vec{w}^{(1)}\right], B_{2}=\left[\vec{v}^{(2)}, \vec{w}^{(2)}\right] \subsetneq \mathbb{Z}^{d}$ with distance $\delta_{\infty}\left(B_{1}, B_{2}\right)>g$, any pair of patterns on $B_{1}$ and $B_{2}$ occurring in $X$ can be put together to form a valid point of $X$, i.e. $\forall P_{1} \in \mathcal{L}_{B_{1}}(X), P_{2} \in \mathcal{L}_{B_{2}}(X) \exists x \in X$ such that $\left.x\right|_{B_{1}}=P_{1}$ and $\left.x\right|_{B_{2}}=P_{2}$ (or equivalently $\forall y, z \in X \exists x \in X$ such that $\left.x\right|_{B_{1}}=\left.y\right|_{B_{1}}$ and $\left.\left.x\right|_{B_{2}}=\left.z\right|_{B_{2}}\right)$.

$\mathrm{A} \mathbb{Z}^{d}$ shift $X$ is called block gluing if it is block gluing at gap $g$ for some $g \in \mathbb{N}_{0}$.

Obviously $\mathbb{Z}^{d}$ full shifts are block gluing at gap $g=0$. Note that a $\mathbb{Z}$ shift is block gluing if and only if it satisfies the specification property [2].

In analogy with the matrix-SFT case in 22, 14 we say that a general $\mathbb{Z}^{d}$ shift $X$ has a safe symbol if its alphabet contains a letter $a$ such that for any $x \in X$, any way of changing some letters in $x$ to as results in a new point still in $X$.

It is well known that every $\mathbb{Z} \mathrm{SFT}$ of entropy at least $\log N$ factors onto the $\mathbb{Z}$ full shift on $N$ symbols. The same holds for $d>1$ in the case of $\mathbb{Z}^{d}$ group shifts 4 . (note that those have entropy $\log N$ for some $N \in \mathbb{N}$ ) but not for general $\mathbb{Z}^{d}$ SFTs [5. 11. Desai proved for $d \geq 2$ that a corner gluing $\mathbb{Z}^{d}$ SFT of entropy strictly greater than $\log N$ factors onto a $\mathbb{Z}^{d}$ full shift on $N$ symbols [9]. (Corner gluing is a condition strictly stronger than block gluing; see Appendix $\mathbf{A}$ ) We prove the following generalization of Desai's theorem.

Theorem 3.2. Suppose $d \geq 1$ and let $X$ be a block gluing $\mathbb{Z}^{d}$ shift. Then the following hold:

(1) If $N \in \mathbb{N}$ and $h(X)>\log N$, then $X$ factors topologically onto the $\mathbb{Z}^{d}$ full shift on $N$ symbols.

(2) X factors topologically onto a family of strongly irreducible $\mathbb{Z}^{d}$ SFTs with entropies dense in $[0, h(X)]$.

(3) X factors topologically onto any lower entropy $\mathbb{Z}^{d}$ SFT having a safe symbol.

Proof. For all three claims we use ideas of Desai's proof of part (1) of our theorem from 9 (stated there under the stronger assumption of $X$ being a corner gluing $\mathbb{Z}^{d}$ SFT), with some adaptations. Due to space considerations, we just sketch our argument here and refer to Desai's paper 9] for finer details.

We may assume $X$ to be block gluing with gap $g \in \mathbb{N}_{0}$ and entropy $h(X)>0$. Fix any $\varepsilon>0$ such that $\varepsilon<h(X)$. Theorem B.2(4) guarantees the existence of a pattern $P \in \mathcal{L}(X)$ such that the subshift $X^{K}:=\{x \in X \mid P$ does not occur in $x\}$ still has entropy $h\left(X^{\nwarrow}\right)>h(X)-\varepsilon$.

Because $h\left(X^{R}\right)>0$, there is a pattern $Q \in \mathcal{L}_{B}\left(X^{\Re}\right)$ on some solid block $B \subsetneq \mathbb{Z}^{d}$ such that for $\vec{\imath} \in \mathbb{Z}^{d}$ with $\|\vec{\imath}\|_{\infty} \leq g+\|P\|_{\infty}$, the pattern $Q$ cannot overlap with a copy of $Q$ translated by $\vec{\imath}$, i.e. $\exists \vec{\jmath} \in B \cap B-\vec{\imath}: Q_{\vec{\jmath}} \neq Q_{\vec{\imath}+\vec{\jmath}}$ (we can use Desai's original construction to show this). Since $X$ is block gluing, there is a solid block pattern $M \in \mathcal{L}(X)$ with $P$ at each corner and also containing $Q$ (see the left part of Figure 11; w.l.o.g. we may assume that $P, Q$, and thus $M$, have the shape of a hypercube.

We define a "surrounded word" to be the central pattern of a configuration on a solid hypercube as pictured in Figure 1. The surrounded words play the role of Desai's "follower words" in the original proof. 


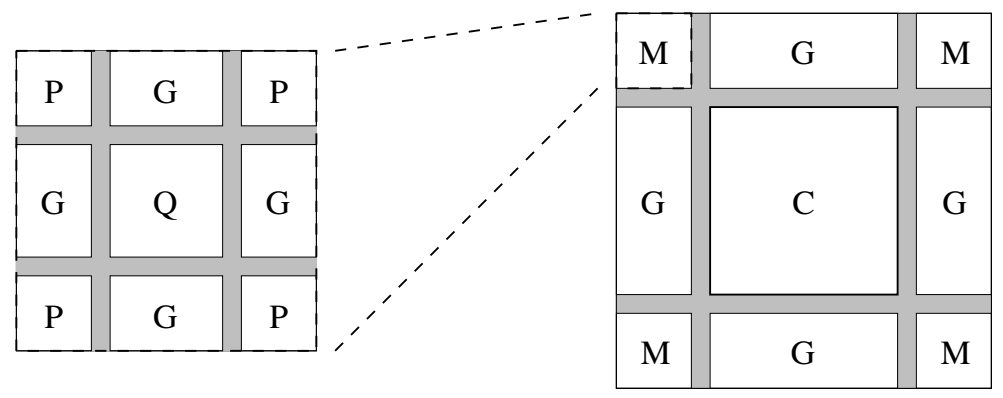

Figure 1. The marker pattern $M$ (left) and a surrounded word $C$ (right).

In Figure 1, each $G$ represents any valid pattern of appropriate shape in which $P$ does not occur, i.e. $G \in \mathcal{L}\left(X^{R}\right)$ (different $G$ symbols may not represent the same word). The unspecified shaded regions represent valid configurations in $X$ (over which we have no control), filling gaps of thickness $g$. By the block gluing property, all possible patterns $C \in \mathcal{L}\left(X^{\nwarrow}\right)$ of appropriate size appear as surrounded words in a configuration in $X$.

To establish claim (1) we choose $\varepsilon:=\frac{1}{2}(h(X)-\log N)>0$ and define a factor map $\phi$ from $X$ to the full shift on symbols $\mathcal{A}:=\{0, \ldots, N-1\}$ which will be defined as suggested by Figure 2, For arbitrarily fixed $n \in \mathbb{N}$ we choose a function $\Phi: \mathcal{L}_{[\overrightarrow{\mathbb{1}}, n \overrightarrow{\mathbb{1}}]}\left(X^{\Re}\right) \rightarrow \mathcal{A}^{[\overrightarrow{\mathbb{1}},(n+r) \overrightarrow{\mathbb{1}}]}$, where $r:=2 g+\|M\|_{\infty}$. If in a point in $X$ we see a configuration with a surrounded word $C \in \mathcal{L}_{[\overrightarrow{\mathbb{1}}, n \overrightarrow{\mathbb{1}}]}\left(X^{\mathrm{R}}\right)$ at coordinates $\vec{\imath}+[\overrightarrow{\mathbb{1}}, n \overrightarrow{\mathbb{1}}]$ $\left(\vec{\imath} \in \mathbb{Z}^{d}\right)$, the image configuration on the solid block $\vec{\imath}-g \overrightarrow{\mathbb{1}}+[\overrightarrow{\mathbb{1}},(n+r) \overrightarrow{\mathbb{1}}] \subsetneq \mathbb{Z}^{d}$ indicated as the larger thicklined region in Figure 2 will be $\Phi(C)$. Wherever this rule does not apply (we do not see a complete surrounded-word-pattern in our coding window), we fill in the image coordinates with 0 s. The map $\phi$ induced by this rule is well defined because the marker structure containing $Q$ ensures that two configurations defining surrounded words can overlap only by the complete overlap of $M$ corners.

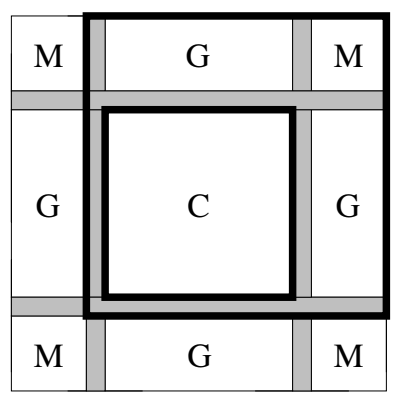

Figure 2. A surrounded word $C$ and the (larger) region it uniquely determines in the $\phi$-image. 
As $h\left(X^{R}\right)>h(X)-\varepsilon=\log N+\varepsilon$ we can fix $n \in \mathbb{N}$ large enough such that $\left|\mathcal{L}_{[\overrightarrow{1}, n \vec{n}]}\left(X^{K}\right)\right|>N^{(n+r)^{d}}$. Thus we may choose $\Phi$ surjective. This implies that $\phi$ is onto: one simply places copies of the pattern $M$ gridding the plane with appropriate distance, and interpolates surrounded words $C$, to produce a specified image point.

For the second part of the theorem we explicitly construct a family of strongly irreducible $\mathbb{Z}^{d}$ SFTs. For any fixed $\varepsilon>0$ we choose $2 \leq m \in \mathbb{N}$ such that $\frac{\log m^{d}}{m^{d}}<\frac{\varepsilon}{2}$. For every $j \in \mathbb{N}$ let $\mathcal{A}_{Y_{j}}:=\{0,1, \ldots,(j-1)\}$ and define $\mathbb{Z}^{d}$ SFTs

$$
Y_{j}:=\left\{y \in \mathcal{A}_{Y_{j}}^{\mathbb{Z}^{d}}\left|\forall \vec{\imath} \in \mathbb{Z}^{d}: y\right|_{\vec{\imath}+[\overrightarrow{\mathbb{1}}, m \overrightarrow{\mathbb{1}}]} \text { contains at most one non-zero symbol }\right\} .
$$

Since there cannot be more than one non-zero symbol in a block of size $[\overrightarrow{\mathbb{1}}, m \overrightarrow{\mathbb{1}}]$, we have $\left|\mathcal{L}_{[\overrightarrow{\mathbb{1}}, m \overrightarrow{\mathbb{1}}]}\left(Y_{j}\right)\right| \leq m^{d} \cdot j$. Using this and the fact that we can place symbols freely on a grid $(m \mathbb{Z})^{d}$ while filling in the remaining coordinates with $0 \mathrm{~s}$, we have

$$
j^{t^{d}} \leq\left|\mathcal{L}_{[\overrightarrow{\mathbb{1}}, t m \overrightarrow{\mathbb{1}}]}\left(Y_{j}\right)\right| \leq\left(m^{d} \cdot j\right)^{t^{d}}
$$

for any $t \in \mathbb{N}$. The entropy of $Y_{j}$ can thus be estimated to be

$$
\frac{\log j}{m^{d}} \leq h\left(Y_{j}\right) \leq \frac{\log j}{m^{d}}+\frac{\log m^{d}}{m^{d}}<\frac{\log j}{m^{d}}+\frac{\varepsilon}{2} .
$$

We claim that each $Y_{j}$ is strongly irreducible with gap $m$. For this let $P_{1} \in$ $\mathcal{L}_{F_{1}}\left(Y_{j}\right), P_{2} \in \mathcal{L}_{F_{2}}\left(Y_{j}\right)$ be two patterns on finite non-empty sets $F_{1}, F_{2} \subsetneq \mathbb{Z}^{d}$ such that $\delta_{\infty}\left(F_{1}, F_{2}\right)>m$. Clearly the points $y^{(1)}, y^{(2)} \in \mathcal{A}_{Y_{j}}^{\mathbb{Z}^{d}}$, defined as $\left.y^{(i)}\right|_{F_{i}}:=P_{i}$ and $y{ }^{(i)} \vec{\imath}:=0$ for every $\vec{\imath} \in \mathbb{Z}^{d} \backslash F_{i}(i=1,2)$, are in $Y_{j}$ (each realizes the pattern $P_{i}$ in a "minimal" way). Suppose the point $y \in \mathcal{A}_{Y_{j}}^{\mathbb{Z}^{d}}$ with $\left.y\right|_{F_{1}}:=P_{1},\left.y\right|_{F_{2}}:=P_{2}$ and $y_{\vec{\imath}}:=0$ for all $\vec{\imath} \in \mathbb{Z}^{d} \backslash\left(F_{1} \cup F_{2}\right)$ is not in $Y_{j}$. Due to the description of $Y_{j}$, there has to be $\vec{\imath} \in \mathbb{Z}^{d}$ such that $\left.y\right|_{\vec{\imath}+[\overrightarrow{\mathbb{1}}, m \overrightarrow{\mathbb{1}}]}$ contains at least two non-zero symbols. But as $y^{(1)}, y^{(2)} \in Y_{j}$, this can only happen if the block $\vec{\imath}+[\overrightarrow{\mathbb{1}}, m \overrightarrow{\mathbb{1}}]$ contains coordinates of both $F_{1}$ and $F_{2}$, which contradicts $F_{1}, F_{2}$ being separated by $m$. So $y \in Y_{j}$ and $Y_{j}$ is strongly irreducible as claimed.

Now choose $j^{*} \in \mathbb{N}$ such that $h(X)-2 \varepsilon<h\left(Y_{j^{*}}\right)<h(X)-\varepsilon$, which is possible by the choice of $m$. Again we find $X^{\Re} \subsetneq X$ with $h\left(X^{\nwarrow}\right)>h(X)-\varepsilon$, and we carry out the marker construction as above. This time we fix $n \in \mathbb{N}$ with $n>m$ large enough that

$$
\frac{\log \left|\mathcal{L}_{[\overrightarrow{\mathbb{1}}, n \overrightarrow{\mathbb{1}}]}\left(X^{\mathrm{R}}\right)\right|}{(n+r)^{d}}>h(X)-\varepsilon>\frac{\log \left|\mathcal{L}_{[\overrightarrow{1},(n+r) \overrightarrow{\mathbb{1}}]}\left(Y_{j^{*}}\right)\right|}{(n+r)^{d}}>h(X)-2 \varepsilon
$$

holds for $r:=2 g+\|M\|_{\infty}$. Hence we have more surrounded words than valid $Y_{j^{*}}$-patterns, and we can choose a surjection $\Phi: \mathcal{L}_{[\overrightarrow{\mathbb{1}}, n \overrightarrow{\mathbb{1}}]}\left(X^{R}\right) \rightarrow \mathcal{L}_{[\overrightarrow{\mathbb{1}},(n+r) \overrightarrow{\mathbb{1}}]}\left(Y_{j^{*}}\right)$ to build our factor map $\phi$. Whenever $\phi$ sees a complete marker configuration on $\vec{\imath}+\left[\left(1-\|M\|_{\infty}\right) \overrightarrow{\mathbb{1}},(n+r) \overrightarrow{\mathbb{1}}\right] \subsetneq \mathbb{Z}^{d}\left(\vec{\imath} \in \mathbb{Z}^{d}\right)$ with a surrounded word $C \in \mathcal{L}_{[\overrightarrow{\mathbb{1}}, n \overrightarrow{\mathbb{1}}]}\left(X^{R}\right)$ in the middle, it tries to apply the rule given by $\Phi$ to place the pattern $\Phi(C)$ at $\vec{\imath}+[\overrightarrow{\mathbb{1}},(n+r) \overrightarrow{\mathbb{1}}]$. To ensure that the image point will be valid for $Y_{j^{*}}$ we have to take care of the local condition defining $Y_{j^{*}}$. Therefore $\phi$ scans a larger neighborhood for surrounded word patterns. If there exists another surrounded word configuration with $C^{\prime} \in \mathcal{L}_{[\overrightarrow{\mathbb{1}}, n \overrightarrow{\mathbb{1}}]}\left(X^{\mathcal{R}}\right)$ in the middle completely contained within a coding window of size $\vec{\imath}+\left[\left(1-\|M\|_{\infty}-n-r-m\right) \overrightarrow{\mathbb{1}},(2 n+2 r+m) \overrightarrow{\mathbb{1}}\right]$ and the image patterns 
$\Phi(C), \Phi\left(C^{\prime}\right)$ are not compatible with the condition on $Y_{j^{*}}, \phi$ just fills in a block of 0s instead. In all other cases $\phi$ puts 0 s, too.

As before, $\phi$ is well defined because of the marker structure, and it is onto since for every point in $Y_{j^{*}}$ we can grid $\mathbb{Z}^{d}$ with markers and fill in appropriate surrounded words (the rule $\Phi$ will always apply, since the image point will satisfy the "non-zero symbols must have distance at least $m$ " condition).

Note that for every $j>1$ there exists a natural factor map $\psi: Y_{j} \rightarrow Y_{j-1}$ (replacing all occurrences of the symbol $(j-1)$ with 0 s), so $X$ factors onto every $Y_{j}$ with $j \in\left\{1,2, \ldots, j^{*}\right\}$. The choice of $m$ implies that the entropies of the finite family of $\mathbb{Z}^{d} \operatorname{SFTs}\left(Y_{j}\right)_{j \in\left\{1,2, \ldots, j^{*}\right\}}$ is a $\varepsilon$-dense set in $[0, h(X)-\varepsilon]$; i.e. $h\left(Y_{1}\right)=0$, $h\left(Y_{j^{*}}\right)>h(X)-2 \varepsilon$ and for $2 \leq j \leq j^{*}$

$$
0 \leq h\left(Y_{j}\right)-h\left(Y_{j-1}\right) \stackrel{\frac{\mathrm{EB}}{<}}{<} \frac{\log j-\log (j-1)}{m^{d}}+\frac{\varepsilon}{2} \leq \frac{\log 2}{m^{d}}+\frac{\varepsilon}{2}<\varepsilon .
$$

As $\varepsilon>0$ was arbitrary we have finished the proof of (2).

For (3) suppose $Y$ is a $\mathbb{Z}^{d}$ SFT with $h(Y)<h(X)$ and safe symbol 0 . The factor map defined for (2) can be used to show that $X$ factors onto $Y$ (we only need the fact that $Y$ is defined by local conditions and replacing symbols with 0 s never violates these constraints). As the argument is almost identical, details are left to the reader.

We do not know a satisfying characterization of the $\mathbb{Z}^{d}$ shifts which factor onto full shifts.

\section{The Restricted Subsystem/Quotient Results}

In this section we will state our main results on the existence of sofic/SFT shifts with restricted subsystems and quotients, and indicate the organization of their proofs in subsequent sections. Recall that by a trivial factor we mean the factor which is the trivial dynamical system consisting of one fixed point.

Theorem 4.1. Given $M>0$ and $d \geq 2$, there exists a $\mathbb{Z}^{d}$ sofic shift $S$ with the following properties.

(1) $h(S)>M$

(2) $S$ (and thus every topological factor of $S$ ) contains a fixed point which is its unique minimal subsystem.

(3) Every topological factor of $S$ (including $S$ ) contains a unique $\mathbb{Z}^{d}$ SFT, which is a fixed point. In particular, $S$ has no non-trivial SFT factor.

(4) If $Y$ is any non-trivial subshift factor of $S$, then $Y$ cannot be block gluing. Hence $Y$ also cannot be strongly irreducible, cannot have the uniform filling property (UFP), and cannot be corner gluing. In particular, $Y$ cannot be a full shift.

(5) In the case $d \geq 3, S$ can be chosen such that for every non-trivial topological factor $Y$ of $S$, there is no invariant Borel probability $\mu \in \mathcal{M}(Y)$ on $Y$ such that $(Y, \mu)$ as a measurable system has completely positive entropy.

(6) $S$ has an equal entropy (sofic) subshift factor of topologically completely positive entropy.

(7) There is a $\mathbb{Z}^{d}$ SFT $X$ and a factor map $\pi: X \rightarrow S$ such that $h(X)=h(S)$, and the preimage in $X$ of the unique fixed point in $S$ is a $\mathbb{Z}^{d} S F T K \subsetneq X$ such that $h(K)=0$. 
(8) In the case $d=2, S$ can in addition be chosen to be mixing and of topologically completely positive entropy.

(9) For $d \geq 3, S$ can be chosen to be mixing and satisfying all the properties (11) -(17) except (6) .

The mixing $S$ we construct to prove Theorem 4.1 in the case $d \geq 3$ has no topological factor which has topologically completely positive entropy.

We have similar results in the case that the subshift must be SFT.

Theorem 4.2. Given $M>0$ and $d \geq 2$, there exists a $\mathbb{Z}^{d}$ SFT $X$ with the following properties.

(1) $h(X)>M$.

(2) X contains a zero entropy SFT $K$ which contains every minimal subsystem of $X$. In particular, every non-empty subsystem of $X$ has to intersect $K$.

(3) If $Y$ is any block gluing shift factor of $X$, then $Y$ is trivial. In particular, the only full shift factor of $X$ is the trivial shift.

(4) If $Y$ is any subshift factor of $X$ and $Y_{\mathrm{MIN}}$ is the orbit closure of its minimal subsystems, then $h\left(Y_{\mathrm{MIN}}\right)=0$.

(5) In the case $d \geq 3, X$ can be chosen such that for every non-trivial topological factor $Y$ of $X$, there is no invariant Borel probability $\mu \in \mathcal{M}(Y)$ on $Y$ such that $(Y, \mu)$ as a measurable system has completely positive entropy.

(6) $X$ has an equal entropy sofic factor with topologically completely positive entropy. In the case $d=2, X$ itself can be chosen to be mixing with topologically completely positive entropy.

Remark 4.3. Our constructions for some claims in the theorems above require $d=2$ or $d \geq 3$. However, we know of no obstruction to the existence of examples for all $d \geq 2$ for which all the claims hold. There is some further give and take in the properties we can demand of the examples within the limitations of our current techniques; for this see Remarks 9.1 and 10.1

Remark 4.4. A $\mathbb{Z}$ sofic shift $S$ contains pairwise disjoint SFTs with entropies dense in $[0, h(S)]$. Desai proved for $d \geq 2$ that a $\mathbb{Z}^{d}$ sofic shift $S$ contains sofic shifts with entropies dense in $[0, h(S)][8$. Theorem 4.1 shows for $d \geq 2$ that we cannot improve Desai's theorem by replacing sofic with SFT or by requiring disjointness.

Similarly, a $\mathbb{Z}$ SFT $X$ contains pairwise disjoint SFTs with entropies dense in $[0, h(X)]$. Desai (generalizing [25]) proved for $d \geq 2$ that a $\mathbb{Z}^{d}$ SFT $X$ contains SFTs with entropies dense in $[0, h(X)]$ [8]. Theorem 4.2 shows for $d \geq 2$ that we cannot improve Desai's theorem by requiring the SFTs to be disjoint.

Remark 4.5. By way of contrast we recall some facts about the subsystems of a mixing $\mathbb{Z}$ SFT $X$. By the Jewett-Krieger Theorem and one of its proofs, every Borel probability preserving measurable system with entropy strictly less than $h(X)$ has a realization as a uniquely ergodic subshift $Y$ of $X[7$. This $Y \subsetneq X$ can be chosen disjoint from any given proper subsystem of $X$ ("there is always room at the Krieger Hotel"). Krieger's Embedding Theorem [15, 19, provides an even more vast array of subsystems. A mixing $\mathbb{Z}$ sofic shift $S$ contains an increasing union of mixing SFTs $X_{n}$ with $\lim _{n \rightarrow \infty} h\left(X_{n}\right)=h(S)$ [21, so these statements also hold for mixing $\mathbb{Z}$ sofic shifts.

Remark 4.6. The abundance of subsystems in $\mathbb{Z}$ SFTs is related to a number of significant coding constructions and in particular to a considerable control over 
their factors. For example [3], if $S$ is a $\mathbb{Z}$ sofic shift and $Y$ is a mixing $\mathbb{Z}$ SFT such that $h(S)>h(Y)$, then $Y$ is a factor of $S$ if and only if

$$
\forall s \in S, n \in \mathbb{N} \text { with } \sigma_{S}^{n}(s)=s \Longrightarrow \exists y \in Y \text { such that } \sigma_{Y}^{n}(y)=y .
$$

Even for $\mathbb{Z}$, when $Y$ above is only sofic the statement fails, and the problem of when there is a factor map between mixing $\mathbb{Z}$ sofic shifts of different entropy is only partly solved; see [3, 29. However, if $X$ is any $\mathbb{Z}$ SFT with $h(X) \geq \log N$, then $X$ factors onto the full $\mathbb{Z}$ shift on $N$ symbols $[3,20$. This led to the following question of Johnson and Madden (see [5] for more motivation).

Question 4.7 (14). If $N \in \mathbb{N}$ is a positive integer and $X$ is a $\mathbb{Z}^{d}$ SFT with entropy $h(X) \geq \log N$, must there exist a continuous factor map from $X$ onto the $\mathbb{Z}^{d}$ full shift on $N$ symbols?

Examples proving a negative answer to Question 4.7 for the case $h(X)=\log N$ and $d \geq 2$ were given in [5] and then [11. Theorem 4.2 proves the negative answer even given a strict entropy inequality.

The proofs of Theorems 4.1 and 4.2 are spread over the next several sections. Sections 5 58 are devoted to the case $d \geq 3$ and are based on a very general subdynamics realization theorem of Hochman [11. Section 5 establishes properties of a $\mathbb{Z}$ shift which is an ingredient for the construction in Section 6. which proves Theorem 4.1 for the case that $d \geq 3$ and $S$ is not mixing. In Section 7 we elaborate this construction to prove Theorem 4.1 for the case that $d \geq 3$ and $S$ is mixing. As a corollary of this construction, in Section 8 we prove Theorem 4.2 for the case $d \geq 3$.

The constructions for $d=2$ do not rely on a general theorem but rather on a specific example from another paper of Hochman [12. In Section 9, we show this example satisfies the conditions of Theorem 4.2 in the case $d=2$. In Section 10 , the case $d=2$ of Theorem 4.1 follows easily. The powerful constructions of $\mathbb{Z}^{d}$ sofic and finite type shifts due to Hochman and Meyerovitch [13 and Hochman 11 (for example, constructing SFTs of all possible entropies and $\mathbb{Z}^{3}$ sofic shifts with every possible one-dimensional subdynamic) have the feature that the constructed systems lie in the class of subshifts which have an infinite, non-mixing, zero entropy factor. This underlines interest in constructions with stronger mixing properties and explains our considerable efforts to achieve topologically mixing examples. The property of topologically completely positive entropy is another step away from the class of Hochman-Meyerovitch examples; on the other hand, the lack of any topological factor supporting a c.p.e. measure for our examples with $d \geq 3$ is a pungent residue of the original zero entropy factor.

\section{Definition of a Certain $\mathbb{Z}$ ESS}

In this section we define a particular $\mathbb{Z}$ shift $Z \subsetneq\{0,1\}^{\mathbb{Z}}$ which will be used to build our $\mathbb{Z}^{d}$ examples when $d \geq 3$. For this choose an integer $N \in \mathbb{N}$ so that $\sum_{n=N+1}^{\infty} n^{-1.1}<1$ and set

$Z:=\left\{z \in\{0,1\}^{\mathbb{Z}} \mid \forall n>N: 0^{n}\right.$ appears in every subword of length $\left\lceil n^{2.1}\right\rceil$ of $\left.z\right\}$.

Recall from 11 that a $d$-dimensional dynamical system is called an effective symbolic system (ESS) if it is conjugate to a $\mathbb{Z}^{d}$ shift $X$ defined over some alphabet $\mathcal{A}$ such that $X$ is given as

$$
X:=X_{\not}=\left\{x \in \mathcal{A}^{\mathbb{Z}^{d}} \mid x \text { does not contain any pattern from } \mathcal{E}\right\},
$$


where the set of excluded patterns $\mathcal{E} \subseteq \mathcal{L}\left(\mathcal{A}^{\mathbb{Z}^{d}}\right)$ is a recursively enumerable set (RE-set), i.e. there exists a Turing machine that given input $n \in \mathbb{N}$ outputs a finite $d$-dimensional pattern $P_{n} \in \mathcal{L}\left(\mathcal{A}^{\mathbb{Z}^{d}}\right)$ over $\mathcal{A}$ such that $\mathcal{E}=\left\{P_{n} \mid n \in \mathbb{N}\right\}$.

Lemma 5.1. The $\mathbb{Z}$ shift $Z$ defined above has the following properties.

(1) $Z$ is topologically mixing.

(2) For every $n \in \mathbb{N}$ the pattern $0^{n}$ appears syndetically in every point in $Z$. Hence the fixed point $0^{\infty}$ is the only minimal subsystem in $Z$.

(3) For any pattern $P \in \mathcal{L}(Z), P$ has positive frequency in some point of $Z$.

(4) $Z$ is an effective symbolic system.

Proof. To prove (1), take any two valid patterns $P_{1}, P_{2} \in \mathcal{L}_{[1, l]}(Z)$ of length $l \in \mathbb{N}$. For any $m \geq \max \{5, l\}$, define a point $z \in\{0,1\}^{\mathbb{Z}}$ by $\left.z\right|_{[1, l]}:=P_{1},\left.z\right|_{[m+l+1, m+2 l]}:=$ $P_{2}$ and $z_{i}:=0$ for all other $i \in \mathbb{Z}$. We claim that $z \in Z$. To see this, we have to check that for every $n>N$ and every subpattern $P$ of $z$ of length $\left[n^{2.1}\right], P$ contains $0^{n}$. We consider two cases: $n \geq m$ and $n<m$.

First suppose $n \geq m$. Using the definition of $m$ this already gives $\left[n^{2.1}\right\rceil>5 n \geq$ $2 n+2 l+m$. A subpattern $P$ of $z$ of length $\left\lceil n^{2.1}\right\rceil$ is at least $2 n+1$ symbols longer than the concatenation $P_{1} 0^{m} P_{2}$ having length $2 l+m$. Therefore $P$ contains more than $n$ consecutive $0 \mathrm{~s}$, either to the left or to the right of $\left.z\right|_{[1, m+2 l]}=P_{1} 0^{m} P_{2}$.

Now assume $n<m$. Choose any subpattern $P$ of $z$ of length $\left[n^{2.1}\right\rceil$. If $P$ contains letters from $P_{1}$ as well as from $P_{2}$, then clearly $P$ covers the gap of $m 0 \mathrm{~s}$ between $P_{1}$ and $P_{2}$, and so contains $0^{n}$. Otherwise $P$ does not contain letters of both patterns, and without loss of generality we may suppose $P$ does not overlap with $P_{2}$. Then $P$ is also a subpattern of the modified point $z^{\prime} \in\{0,1\}^{\mathbb{Z}}$, defined by $\left.z^{\prime}\right|_{[1, l]}:=P_{1}$ and $z_{i}:=0$ for all other $i \in \mathbb{Z}$. Since $P_{1} \in \mathcal{L}_{[1, l]}(Z)$, it occurs in some $z^{\prime \prime} \in Z$ (with $\left.z^{\prime \prime}\right|_{[1, l]}=P_{1}$ ). Note that every letter of $z^{\prime}$ is less than or equal to the corresponding letter of $z^{\prime \prime}$. If $P=\left.z^{\prime}\right|_{\left[j, j+\left\lceil n^{2.1}\right\rceil-1\right]}$ for some $j \in \mathbb{Z}$, then define $P^{\prime}:=\left.z^{\prime \prime}\right|_{\left[j, j+\left\lceil n^{2.1}\right\rceil-1\right]}$. As $P^{\prime}$ is a subpattern of $z^{\prime \prime} \in Z$ of length $\left\lceil n^{2.1}\right\rceil$, it has to contain $0^{n}$. Finally, $P$ occupying the same position in $z^{\prime}$ as $P^{\prime}$ in $z^{\prime \prime}$ has to be coordinatewise less than or equal to $P^{\prime}$, and thus $P$ contains $0^{n}$ as well.

Therefore any subpattern of $z$ of length $\left[n^{2.1}\right]$ contains $0^{n}$, and we have shown that $z \in Z$. As $P_{1}, P_{2}$ were chosen arbitrarily, $Z$ is (topologically) mixing.

The definition of $Z$ trivially implies (2).

To prove (3), it clearly suffices to consider legal patterns $P_{0} \in \mathcal{L}_{\left[1, l_{0}\right]}(Z)$ of big length, e.g. $l_{0}>5^{20}$. The proof of (1) demonstrates that for $m_{0} \in \mathbb{N}$ such that the inequality $\left[n^{2.1}\right]>2 n+2 l_{0}+m_{0}$ holds for every $n \geq m_{0}$, the point $z \in\{0,1\}^{\mathbb{Z}}$ defined by $\left.z\right|_{\left[1, l_{0}\right]}:=\left.z\right|_{\left[m_{0}+l_{0}+1, m_{0}+2 l_{0}\right]}:=P_{0}$ and $z_{i}:=0$ for all other $i \in \mathbb{Z}$ is in $Z$. This condition is satisfied by any $m_{0}>\sqrt{l_{0}}$, since for such $m_{0}$ and any $n \geq m_{0}$ we get $\left\lceil n^{2.1}\right\rceil>5 n^{2}>2 n^{2}+3 n>2 n+2 m_{0}^{2}+m_{0}>2 n+2 l_{0}+m_{0}$. In particular, the word $P_{1}:=P_{0} 0^{m_{0}} P_{0}$ is a legal pattern in $Z$ for $m_{0}:=2\left\lfloor\sqrt{l_{0}}\right\rfloor$. Denote by $l_{1} \in \mathbb{N}$ the length of $P_{1}$. Then by the same argument, for $m_{1}:=2\left[\sqrt{l_{1}}\right]$, the word $P_{2}:=P_{1} 0^{m_{1}} P_{1}$ is a legal pattern in $Z$. Continuing in this fashion we inductively define a sequence $\left(P_{n} \in \mathcal{L}_{\left[1, l_{n}\right]}(Z)\right)_{n \in \mathbb{N}_{0}}$, where $P_{n+1}:=P_{n} 0^{2}\left\lfloor\sqrt{l_{n}}\right\rfloor P_{n}$, and $l_{n+1}:=2 l_{n}+2\left\lfloor\sqrt{l_{n}}\right\rfloor$. Since $P_{n}$ is a prefix of $P_{n+1}$ for every $n \in \mathbb{N}_{0}$, there is a point $z \in Z$ so that $\left.z\right|_{\left[0, l_{n}-1\right]}=P_{n}$ for all $n \in \mathbb{N}_{0}$. We claim that the frequency of $P_{0}$ in the point $z$ is positive. 
Define the sequence $\left(r_{n}:=\frac{l_{n}}{2^{n}}\right)_{n \in \mathbb{N}_{0}}$. Then, $r_{0}=l_{0}$, and for $n \in \mathbb{N}_{0}$ we get $r_{n+1}=\frac{2 l_{n}+2\left\lfloor\sqrt{l_{n}}\right\rfloor}{2^{n+1}}=\frac{l_{n}+\left\lfloor\sqrt{l_{n}}\right\rfloor}{2^{n}} \leq r_{n}+2^{-\frac{n}{2}} \sqrt{r_{n}}$. For large $n$ we immediately get a loose but sufficient estimate $r_{n+1}<1.5 r_{n}$. So we can assume $r_{n}<1.62^{n}$ at least for $n$ big enough. This yields $r_{n+1} \leq r_{n}+2^{-\frac{n}{2}} \sqrt{r_{n}}<r_{n}+2^{-\frac{n}{2}} \sqrt{1.62^{n}}=r_{n}+0.9^{n}$ again for $n$ large. Applying the geometric series, the strictly increasing sequence $\left(r_{n}\right)_{n \in \mathbb{N}_{0}}$ has a positive limit, call it $r \in \mathbb{R}^{+}$. Note that by definition, $P_{0}$ appears at least $2^{n}$ times in $\left.z\right|_{\left[0, l_{n}-1\right]}=P_{n}$ for all $n \in \mathbb{N}_{0}$. Therefore, the frequency $f_{P_{0}}(z) \in \mathbb{R}$ of $P_{0}$ in $z$ is at least

$$
f_{P_{0}}(z)=\lim _{n \rightarrow \infty} \frac{\# P_{0} \mathrm{~s} \text { in }\left.z\right|_{\left[-l_{n}, l_{n}\right]}}{2 l_{n}+1} \geq \lim _{n \rightarrow \infty} \frac{2^{n}}{2 l_{n}\left(1+\frac{1}{2 l_{n}}\right)}=\lim _{n \rightarrow \infty} \frac{1}{2 r_{n}}=\frac{1}{2 r}>0,
$$

proving (3).

To get (4), we have to find an RE-set $\mathcal{E} \subseteq \mathcal{L}\left(\{0,1\}^{\mathbb{Z}}\right)$ of excluded patterns such that

$Z=\left\{z \in\{0,1\}^{\mathbb{Z}} \mid z\right.$ does not contain any subpattern which is an element of $\left.\mathcal{E}\right\}$.

By simply rewriting the definition of $Z$, we observe that the following set will do:

$$
\mathcal{E}:=\bigcup_{n=N+1}^{\infty}\left\{P \in\{0,1\}^{\left[1,\left\lceil n^{2.1}\right\rceil\right]} \mid P \text { does not contain } 0^{n}\right\} .
$$

It is not difficult to design an algorithm to construct the elements of $\mathcal{E}$ in (lengthlexicographic) order. Therefore, $\mathcal{E}$ is a recursively enumerable set, and so $Z$ is an effective symbolic system.

The $\mathbb{Z}$ shift $Z$ will be used as a main ingredient in the constructions in the following three sections.

\section{The NON-MiXING $\mathbb{Z}^{d}$ SOFIC EXAMPLES For $d \geq 3$}

In this section, we prove Theorem 4.1 in the case that $d \geq 3$ and the constructed sofic shift is not required to be mixing.

Proof. Let $Z$ be the ESS obtained in Lemma 5.1] For each integer $1 \leq i \leq d$ define the $\mathbb{Z}^{d}$ shift $T_{i}$ as the set of all configurations $t \in\{0,1\}^{\mathbb{Z}^{d}}$ satisfying the following:

$$
\begin{aligned}
& \left(t_{n \vec{e}_{i}}\right)_{n \in \mathbb{Z}} \in Z \text { and } \\
& t_{\vec{\imath}}=t_{\vec{\imath}+\vec{e}_{j}} \quad \forall \vec{\imath} \in \mathbb{Z}^{d} \text { and } j \neq i .
\end{aligned}
$$

So, along the axis $\mathbb{Z} \vec{e}_{i}$, a point $t$ in $T_{i}$ is a copy of a point in $Z$; in perpendicular directions, $t$ just copies symbols from that axis. Because $d \geq 3$ and $Z$ is a $\mathbb{Z}$ ESS, Hochman's ESS subdynamics realization theorem [11, together with its proof, shows that $T_{i}$ is a $\mathbb{Z}^{d}$ sofic shift, such that for each $1 \leq i \leq d$ there is a one-block factor map $\gamma_{i}: V_{i} \rightarrow T_{i}$, where $V_{i}$ is a zero entropy $\mathbb{Z}^{d} \mathrm{SFT}$.

Next we map the $\mathbb{Z}^{d}$ sofic shift $T_{1} \times T_{2} \times \cdots \times T_{d}$ onto a sofic shift $S^{\prime} \subsetneq\{0,1\}^{\mathbb{Z}^{d}}$ by the one-block code defined by the rule sending a symbol $\left(a_{1}, a_{2}, \ldots, a_{d}\right) \in\{0,1\}^{d}$ to the product $a_{1} a_{2} \cdots a_{d}$. This product is 0 unless every $a_{i}$ is 1 . Note that $S^{\prime}$ has entropy zero. Finally, we will pick $m \in \mathbb{N}$ and define $S$ by freely splitting the symbol 1 of $S^{\prime}$ into $m$ symbols $1_{1}, 1_{2}, \ldots, 1_{m}$; i.e. a point $s$ is in $S$ if and only if its image under the one-block factor map $\kappa: S \rightarrow S^{\prime}$ replacing all the $1_{j} \mathrm{~s}(1 \leq j \leq m)$ with 1 is in $S^{\prime}$. 
By Lemma [5.1] the ESS $Z$ has the following property: every allowed finite configuration occurs with positive frequency in some point of the subshift. This property is inherited by the product $T_{1} \times T_{2} \times \cdots \times T_{d}$, then by the factor $S^{\prime}$, and then by $S$. As in [13, the entropy of $S$ is $\log m$ multiplied by the maximum frequency of the symbol 1 in a point of $S^{\prime}$, which we have noted is positive. We fix $m \in \mathbb{N}$ so that $h(S)>M$, establishing (1).

We have an obvious one-block factor map from the zero entropy $\mathbb{Z}^{d}$ SFT $X^{\prime}:=$ $V_{1} \times V_{2} \times \cdots \times V_{d}$ onto $S^{\prime}$, which we denote by $\pi^{\prime}: X^{\prime} \rightarrow S^{\prime}$. We define a $\mathbb{Z}^{d}$ SFT $X$ by freely splitting each preimage symbol $\tilde{1} \in \pi^{\prime-1}(1)$ of 1 in $X^{\prime}$ into $m$ symbols $\tilde{1}_{1}, \ldots, \tilde{1}_{m}$, and then define a one-block factor map $\pi: X \rightarrow S$ by sending each $\tilde{1}_{j}$ to $1_{j}(1 \leq j \leq m)$ and otherwise using the one-block rule for $\pi^{\prime}$. Clearly $h(X)=h(S)$, and the preimage $K:=\pi^{-1}\left(0^{\mathbb{Z}^{d}}\right)$ of the fixed point $0^{\mathbb{Z}^{d}} \in S$ has entropy zero. Because $0^{\mathbb{Z}^{d}}$ and $X$ are SFTs, $K$ is an SFT, and (7) follows.

By construction, arbitrarily long blocks of 0s appear syndetically in all points of the one-dimensional ESS $Z$ (Lemma 5.1(2)). This, together with the periodicity constraints $(\mathrm{PC})$, forces the syndetic occurrence of $(d-1)$-dimensional hyperplanes of $0 \mathrm{~s}$ of any fixed "thickness" (perpendicular to the $\vec{e}_{i}$-axis) in points of $T_{i}$. Points from the factor system $S^{\prime}$ then have a gridlike structure, where the walls of the grid consist of $(d-1)$-dimensional hyperplanes of 0 s separating solid blocks of $1 \mathrm{~s}$. In particular, arbitrarily large $d$-dimensional hypercubes of 0 s occur in every point, and thus the fixed point $0^{\mathbb{Z}^{d}}$ is the only minimal subsystem of $S$. Consequently any factor of $S$ has as its unique minimal subsystem a fixed point, proving (2).

Moreover, for any $n \in \mathbb{N}$ and any finite pattern $P \in \mathcal{L}_{F}(S)\left(F \subsetneq \mathbb{Z}^{d}\right.$ finite) occuring in a point $s \in S$, the pattern $P$ must occur in $s$ inside a pattern $P^{\prime}:=\left.s\right|_{B}$ on some larger block $B \supseteq F$ for which all sites within distance $n$ of the boundary are occupied by 0 s. If an SFT inside $S$ contains a point $s$ other than $0^{\mathbb{Z}^{d}}$, then we can find in $s$ a non-zero pattern $P^{\prime}$ of this form, with $n$ large enough (larger than the order of the SFT), thus a tiling of $\mathbb{Z}^{d}$ with $P^{\prime}$ would produce a legal, non-zero periodic point. This is impossible since $0^{\mathbb{Z}^{d}}$ is the unique minimal subsystem of $S$. The gridlike structure of $0 \mathrm{~s}$ is inherited by any $\mathbb{Z}^{d}$ shift factor of $S$, proving (3) for subshift factors of $S$. If there were a factor map $\phi$ from $S$ onto any compact metrizable system containing a subsystem isomorphic to a non-trivial $\mathbb{Z}^{d} \mathrm{SFT} Y$, the restriction of $\phi$ to $\phi^{-1}(Y)$ would be isomorphic to a block code, which we could extend to $S$ to realize an isomorphic copy of $Y$ in some subshift factor of $S$, which is impossible. This proves (3).

If a non-trivial factor of $S$ were block gluing, by Theorem B.2 it would contain a subsystem disjoint from the image of the fixed point $0^{\mathbb{Z}^{d}}$, contradicting (2). This proves (44). Statement (5) follows from Proposition 6.1, as any point in $S^{\prime}$ contains arbitrarily large hypercubes of $0 \mathrm{~s}$, and hence condition 6.1(2) is satisfied for $W=$ $\left\{0^{\mathbb{Z}^{d}}\right\}$ being the fixed point of all 0 s.

Finally we construct an explicit factor $U$ of $S$ satisfying (6). We define $U$ as the image of $S$ under the one-block map $\psi$ which replaces $1_{1}$ with 0 and changes no other symbol. The (one-block) injective map $\psi \times \kappa: S \rightarrow U \times S^{\prime}$ has a (one-block) inverse. Thus $h(U) \leq h(S) \leq h(U)+h\left(S^{\prime}\right)=h(U)$. This implies $h(S)=h(U)$.

Now suppose there is a factor map $\phi: U \rightarrow Y$ onto a non-trivial subshift $Y$. Let $\widetilde{0}$ and $\widetilde{1}$ be distinct symbols in the alphabet of $Y$ such that $\widetilde{0}^{\mathbb{Z}^{d}}$ is the unique fixed point of $Y$. Let $B:=[-n \overrightarrow{\mathbb{1}}, n \overrightarrow{\mathbb{1}}]$, where $n \in \mathbb{N}$ is chosen large enough that for 
all $s \in S,\left.s\right|_{B}$ determines $(\phi(\psi s))_{0}$. Choose $s \in S$ such that $(\phi(\psi s))_{0}=\widetilde{1}$ and let $Q$ be the block $\left.s\right|_{B}$. Note that $Q$ contains at least one of the symbols $1_{j}, j>1$.

Let $s$ be a point in $S$ in which $Q$ occurs with positive frequency. Thus there is a subset $\mathcal{C} \subseteq \mathbb{Z}^{d}$ of positive density in $\mathbb{Z}^{d}$ such that for all $\vec{\imath} \in \mathcal{C}$ the restriction of $s$ to $\vec{\imath}+B$ is $Q$. Perhaps after reducing the frequency, we may further assume these translates of $B$ by the vectors $\vec{\imath}$ are pairwise disjoint. Let $Q_{1}$ be the pattern which equals $Q$, except that every $1_{j}$ with $j>1$ is replaced with $1_{1}$. If $\left.s\right|_{B}=Q_{1}$, then $(\phi(\psi s))_{0}=\widetilde{0}$. The block $Q_{1}$ can be substituted in $s$ for $Q$ at $\vec{\imath}+B$ for any subset of vectors $\vec{\imath}$ from $\mathcal{C}$ to produce a legal point of $S$. Consequently, in $Y$ all possible patterns of $\widetilde{0}$ and $\widetilde{1}$ on $\mathcal{C}$ have to appear. This implies that $Y$ has positive entropy, and finishes the proof of the non-mixing and $d \geq 3$ case of Theorem 4.1.

Proposition 6.1. Suppose $S$ is a $\mathbb{Z}^{d}$ action by homeomorphisms on a compact metric space $X$ such that the following hold.

(1) There exists a topological factor map $\kappa:(X, S) \rightarrow\left(X^{\prime}, S^{\prime}\right)$ such that $\left(X^{\prime}, S^{\prime}\right)$ is infinite and has zero entropy.

(2) There exists a zero entropy subsystem $(W, S \mid W)$ of $(X, S)$ such that for every $x^{\prime}$ in $X^{\prime}$ there is a sequence $\left(\vec{\imath}_{n}\right)_{n \in \mathbb{N}}$ of vectors in $\mathbb{Z}^{d}$ such that the sequence $S^{\vec{r}_{n}} \kappa^{-1}\left(x^{\prime}\right)$ accumulates on $W$.

Then no non-trivial topological $\mathbb{Z}^{d}$ factor $(Y, T)$ of $(X, S)$ admits an invariant probability $\mu \in \mathcal{M}_{T}(Y)$, such that $(Y, T, \mu)$ as a measurable system has completely positive entropy.

Proof. Let $\phi:(X, S) \rightarrow(Y, T)$ be a continuous factor map, and suppose $(Y, T, \mu)$ is c.p.e. Let $\widetilde{\mu} \in \mathcal{M}_{S}(X)$ be an $S$-invariant probability such that $\phi \widetilde{\mu}=\mu$, and let $\mu^{\prime}:=\kappa \widetilde{\mu}$. We have factor maps of $\mathbb{Z}^{d}$ measure-preserving systems, $\kappa:(X, S, \widetilde{\mu}) \rightarrow$ $\left(X^{\prime}, S^{\prime}, \mu^{\prime}\right)$ and $\phi:(X, S, \widetilde{\mu}) \rightarrow(Y, T, \mu)$. Because one factor is zero entropy and the other is c.p.e., the disjointness theorem of 10 tells us that the map $\kappa \times \phi$ sends $\widetilde{\mu}$ onto the product measure $\mu^{\prime} \times \mu$.

Now consider the decomposition of $\widetilde{\mu}$ relative to $\mu^{\prime}$, which for $\mu^{\prime}$-almost all $x^{\prime}$ in $X^{\prime}$ assigns a measure $\widetilde{\mu}_{x^{\prime}}$ to the fiber $\kappa^{-1}\left(x^{\prime}\right)$. The family of measures $(\kappa \times \phi) \widetilde{\mu}_{x^{\prime}}$ on the sets $\left\{x^{\prime}\right\} \times Y$ give a decomposition of $\mu^{\prime}$ relative to the product measure $\mu^{\prime} \times \mu$. Therefore, on an $S$-invariant set of full $\widetilde{\mu}$ measure, $\phi$ must send $\widetilde{\mu}_{x^{\prime}}$ to $\mu$. By (2), we may choose a sequence $\left(\nu_{n}\right)_{n \in \mathbb{N}}$ of such measures, $\nu_{n}:=\widetilde{\mu}_{x^{\prime}(n)}$, which as measures on the compact metric space $X$ converge to a measure $\nu$ supported on the subset $W$. ( $S$-invariance of the measures $\nu_{n}$ is neither available nor needed.) Because $\phi \nu_{n}=\mu$ for all $n \in \mathbb{N}$ and $\phi$ is continuous, it follows that $\phi \nu=\mu$. This means that the support of $\mu$ is contained in $\phi(W)$. Because the restriction of $T$ to $\phi(W)$ has zero entropy, this contradicts c.p.e. and proves the proposition.

\section{The MiXing $\mathbb{Z}^{d}$ SOFIC EXAMPles For $d \geq 3$}

Elaborating the construction of Section [6] in this section we will construct a family of topologically mixing $\mathbb{Z}^{d}$ sofic shifts $\widetilde{S}$ to establish Theorem 4.1 for $d \geq 3$ in the mixing case.

The construction of $\widetilde{S}$ borrows from the ribbon shift from [24], the technique of "transmitting information" over long distances in a $\mathbb{Z}^{d}$ SFT used in [11] and [13, and the results about SFTs mapping onto multi-dimensional substitution systems from [23]. Begin with the sofic shifts $T_{i}$ defined in the previous section, i.e. $T_{i}$ contains the $\mathbb{Z}$ ESS $Z$ as its one-dimensional subdynamics in the $\vec{e}_{i}$-direction, and 
$T_{i}$ is periodic with respect to $\vec{e}_{j}$ for all $1 \leq j \neq i \leq d$. Each $T_{i}$ has an SFT cover $V_{i}$. Recall that each $V_{i}$ is a nearest neighbor shift, i.e. the forbidden patterns in $V_{i}$ are just pairs of adjacent symbols in any $\vec{e}_{j}$-direction $(1 \leq j \leq d)$, and that each symbol carries enough information to determine the corresponding symbol in the sofic factor $T_{i}$. Denote by $\gamma_{i}$ the one-block factor map from $V_{i}$ to $T_{i}$, and denote by $\mathcal{A}_{i}$ the alphabet of $V_{i}$.

For any $1 \leq i \leq d$, define a new SFT $W_{i}$ as follows. The alphabet for $W_{i}$ is $\mathcal{A}_{i} \dot{\cup} \mathcal{A}_{i}^{\prime}$, where each symbol of $\mathcal{A}_{i}^{\prime}:=\left\{\left.\right|_{a} \mid a \in \mathcal{A}_{i}\right\}$ is a line in the $\vec{e}_{i}$-direction - which from now on, we will assume to be vertical - labeled with a symbol from $\mathcal{A}_{i}$. We will call the elements of $\mathcal{A}_{i}^{\prime}$ vertical line segments, and the terms "below" and "above" will be used to refer to adjacency in the $\vec{e}_{i}$-direction. We will put constraints on the transitions between elements of $\mathcal{A}_{i} \dot{\cup} \mathcal{A}_{i}^{\prime}$ with the effect that points in $W_{i}$ contain "gently ascending hyperplanes" of elements of $\mathcal{A}_{i}$ which are separated vertically by one or more vertical line segments from $\mathcal{A}_{i}^{\prime}$.

We begin with the exact definition of the allowed adjacencies. In points of $W_{i}$, if a symbol $b \in \mathcal{A}_{i}$ appears at coordinate $\vec{\imath} \in \mathbb{Z}^{d}$, then for any $k \neq i$, a symbol $c \in \mathcal{A}_{i}$ must appear at $\vec{\imath}+\vec{e}_{k}$ or $\vec{\imath}+\vec{e}_{k}+\vec{e}_{i}$, but not in both places. We say that the rise in direction $\vec{e}_{k}$ from $\vec{\imath}$ is 0 in the former case and 1 in the latter case. Similarly, a symbol $a \in \mathcal{A}_{i}$ must appear at either $\vec{\imath}-\vec{e}_{k}$ or $\vec{\imath}-\vec{e}_{k}-\vec{e}_{i}$, but not in both places. In any of these cases, the pairs of symbols $b c$ and $a b$ must be legal patterns in the $\vec{e}_{k}$-direction in $V_{i}$. We also impose a homogeneity constraint on the rise: whenever $i \neq j \neq k \neq i$, and a symbol of $\mathcal{A}_{i}$ appears at coordinate $\vec{\imath} \in \mathbb{Z}^{d}$, we require that the rise in direction $\vec{e}_{k}$ from $\vec{\imath}$ equals the rise in direction $\vec{e}_{k}$ from $\vec{\imath}+\vec{e}_{j}$, resp. $\vec{\imath}+\vec{e}_{j}+\vec{e}_{i}$. (Note that rises in different directions add up; i.e. if there is a rise of 1 from the $\vec{e}_{k}$ direction and also from the $\vec{e}_{j}$ direction, then going from coordinate $\vec{\imath}$ to $\vec{\imath}+\vec{e}_{k}+\vec{e}_{j}$, the $\vec{e}_{i}$ coordinate of the ascending hyperplane increases by 2.)

We go on to complete the adjacency rules. If $a \in \mathcal{A}_{i}$ appears at coordinate $\vec{\imath}$, then the vertical line segment $\left.\right|_{a} \in \mathcal{A}_{i}^{\prime}$ must appear at $\vec{\imath}-\vec{e}_{i}$, and some symbol $\left.\right|_{b} \in \mathcal{A}_{i}^{\prime}$ must appear at $\vec{\imath}+\vec{e}_{i}$, where the pair $a b$ must be a legal pattern in the $\vec{e}_{i}$-direction in $V_{i}$. Above a vertical line segment $\left.\right|_{a} \in \mathcal{A}_{i}^{\prime}$ we can have either another $\left.\right|_{a} \in \mathcal{A}_{i}^{\prime}$ or the symbol $a \in \mathcal{A}_{i}$, but nothing else. Hence putting $a \in \mathcal{A}_{i}$ at coordinate $\vec{\imath}$, at position $\vec{\imath}+\vec{e}_{k}$, we have to see either $b \in \mathcal{A}_{i}$ or $\left.\right|_{b} \in \mathcal{A}_{i}^{\prime}$ with the pattern $a b$ being valid in the $\vec{e}_{k}$-direction in $V_{i}$ for the chosen $k \neq i$. Finally, if we see an element $\left.\right|_{a} \in \mathcal{A}_{i}^{\prime}$ at $\vec{\imath}$ we can always use a vertical line segment $\left.\right|_{b} \in \mathcal{A}_{i}^{\prime}$ for which $a b$ is a valid pattern in the $\vec{e}_{k}$-direction in $V_{i}$ to fill coordinate $\vec{\imath}+\vec{e}_{k}$. However, in addition to this possibility we may also put there a symbol $d \in \mathcal{A}_{i}$, whenever the coordinate $\vec{\imath}-\vec{e}_{i}$ contains an element of $\mathcal{A}_{i}$, say $c \in \mathcal{A}_{i}$ for which $c d$ again has to be a valid pattern in the $\vec{e}_{k}$-direction in $V_{i}$.

We define a gently ascending hyperplane in a point $w$ in $W_{i}$ to be a subset $H$ of $\mathbb{Z}^{d}$ such that $w_{\vec{\imath}} \in \mathcal{A}_{i}$ for all $\vec{\imath} \in H$, and $H$ is a maximal connected set under paths built out of elementary paths $(\vec{\imath}, \vec{\jmath})$ such that both $\vec{\imath}$ and $\vec{\jmath}$ are in $H$ and $\vec{\jmath}-\vec{\imath}= \pm\left(\vec{e}_{k}+\Delta \vec{e}_{i}\right)$ for some $k \neq i$ and some $\Delta \in\{0,1\}$. The homogeneity constraint guarantees that orthogonal projection along $\vec{e}_{i}$ from a gently ascending hyperplane to $\left\{\vec{\jmath} \in \mathbb{Z}^{d} \mid \vec{\jmath}_{i}=0\right\}$ is a bijection. The definition of gently ascending hyperplanes allows them to ascend upwards with a local slope in cardinal directions not exceeding 1.

Note that for every $w$ in $W_{i}$ and every $\vec{\imath} \in \mathbb{Z}^{d}$ the vertical axis $\vec{\imath}+\mathbb{Z} \vec{e}_{i}$ intersects every gently ascending hyperplane in $w$ exactly once. The restriction of $w$ to these 
intersections, read consecutively, gives a legal configuration for $Z$ (which could be empty, finite, half-infinite or bi-infinite). This configuration determines the $\mathcal{A}_{i}^{\prime}$ symbols of the remaining sites on the axis, except perhaps in the case that the set $\left\{n \in \mathbb{Z} \mid \vec{\imath}+n \vec{e}_{i} \in H\right.$, for some gently ascending hyperplane $\left.H\right\}$ is bounded above.

The effect of these adjacency rules is illustrated in Figure 3. The left half is a portion of a potential point from $W_{i}\left(\vec{e}_{i}\right.$ is in the vertical direction); the right half is the corresponding word in $V_{i}$ which must be legal in $V_{i}$ in order for the left half to be legal in $W_{i}$. Rows on the right correspond to gently ascending hyperplanes on the left, and the portions between the gently ascending hyperplanes on the left are filled with letters of $\mathcal{A}_{i}^{\prime}$ whose values are forced. The reader should note a slight issue which has to be addressed: our construction of the ascending hyperplanes has only been defined for $d \geq 3$; however Figure 3 shows $d=2$ dimensions. Nevertheless one can consider the picture as a cross-section of a similar picture for $d \geq 3$. The extra issue which appears for $d \geq 3$ but which cannot be expressed in a two-dimensional picture is the homogeneity constraint on the rises as described above. This is largely technical and should not pose a problem to understanding as long as the reader is aware of it.

$\begin{array}{cccccccc}\left.\right|_{\mathrm{c}} & \left.\right|_{\mathrm{b}} & \left.\right|_{\mathrm{c}} & \left.\right|_{\mathrm{a}} & \mathbf{a} & \mathbf{b} & \mathbf{a} & \mathbf{a} \\ \mathbf{a} & \mathbf{b} & \mathbf{c} & \mathbf{b} & \left.\right|_{\mathrm{a}} & \left.\right|_{\mathrm{b}} & \left.\right|_{\mathrm{a}} & \left.\right|_{\mathrm{a}} \\ \left.\right|_{\mathrm{a}} & \left.\right|_{\mathrm{b}} & \left.\right|_{\mathrm{c}} & \left.\right|_{\mathrm{b}} & \left.\right|_{\mathrm{a}} & \mathbf{b} & \mathbf{a} & \mathbf{b} \\ \left.\right|_{\mathrm{a}} & \left.\right|_{\mathrm{b}} & \mathbf{a} & \mathbf{b} & \mathbf{a} & \left.\right|_{\mathrm{b}} & \left.\right|_{\mathrm{a}} & \left.\right|_{\mathrm{b}} \\ \mathbf{a} & \mathbf{b} & \left.\right|_{\mathrm{a}} & \left.\right|_{\mathrm{b}} & \left.\right|_{\mathrm{a}} & \left.\right|_{\mathrm{b}} & \left.\right|_{\mathrm{a}} & \mathbf{a} \\ \left.\right|_{\mathrm{a}} & \left.\right|_{\mathrm{b}} & \left.\right|_{\mathrm{a}} & \left.\right|_{\mathrm{b}} & \left.\right|_{\mathrm{a}} & \mathbf{c} & \mathbf{b} & \left.\right|_{\mathrm{a}}\end{array}$

$\begin{array}{llllllll}\mathrm{c} & \mathrm{b} & \mathrm{c} & \mathrm{a} & & & & \\ \mathrm{a} & \mathrm{b} & \mathrm{c} & \mathrm{b} & \mathrm{a} & \mathrm{b} & \mathrm{a} & \mathrm{a} \\ \mathrm{a} & \mathrm{b} & \mathrm{a} & \mathrm{b} & \mathrm{a} & \mathrm{b} & \mathrm{a} & \mathrm{b} \\ & & & & & \mathrm{c} & \mathrm{b} & \mathrm{a}\end{array}$

Figure 3. A portion of a point from $W_{i}$ (left) and the portion of a point from $V_{i}$ (right) which induces it.

The sofic shift $U_{i} \subsetneq\{0,1\}^{\mathbb{Z}^{d}}$ is obtained by applying the one-block factor map $\varpi_{i}: W_{i} \rightarrow U_{i}$, where $\varpi_{i}(a):=\gamma_{i}(a)$ for $a \in \mathcal{A}_{i}$ and $\varpi_{i}\left(\left.\right|_{a}\right):=0$ for any $\left.\right|_{a} \in \mathcal{A}_{i}^{\prime}$ (in order to keep notation simple we will no longer distinguish between a one-block factor map acting on points of a subshift and the corresponding maps acting on the level of valid patterns or even symbols). By a gently ascending hyperplane in $U_{i}$ we mean the image of a gently ascending hyperplane in $W_{i}$. Roughly speaking, flat hyperplanes of $1 \mathrm{~s}$ in a point of $V_{i}$ give rise to gently ascending hyperplanes of $1 \mathrm{~s}$ in $U_{i}$, separated vertically by some positive number of $0 \mathrm{~s}$.

In a second step we actually have to change $W_{i}$ and $U_{i}$ slightly to construct the objects with which we will want to work. The problem with $U_{i}$ is that there is too much choice where hyperplanes are allowed to ascend, which will both ruin the grid structure used heavily in Section 6 and cause $U_{i}$ to already have positive entropy, rendering the proof of part (5) of Theorem 4.1 unusable. We will solve this by superimposing a point from a $\mathbb{Z}^{d-1}$ SFT over each ascending hyperplane, which tells the hyperplane when it is possible to ascend one step and allows ascensions only very rarely. This restrictive SFT is made by using a result of Mozes about 
substitution dynamical systems in $\mathbb{Z}^{d}$ for $d \geq 2$, for which we must give some definitions.

Definition 7.1. A $\mathbb{Z}^{d}$ substitution rule is a map $\tau: \mathcal{A} \rightarrow \mathcal{A}^{[\overrightarrow{\mathbb{1}}, n \overrightarrow{\mathbb{1}}]}$ for some integer $n>1$. For any $m>1, \tau$ can also be thought of as a map from $\mathcal{A}^{[\overrightarrow{\mathbb{1}}, m \overrightarrow{\mathbb{1}}]}$ to $\mathcal{A}^{[\overrightarrow{\mathbb{1}}, m n \overrightarrow{\mathbb{1}}]}$ by identifying $\mathcal{A}^{[\overrightarrow{\mathbb{1}}, m n \overrightarrow{\mathbb{1}}]}$ with $\left(\mathcal{A}^{[\overrightarrow{\mathbb{1}}, n \overrightarrow{\mathbb{1}}]}\right)^{[\overrightarrow{\mathbb{1}}, m \overrightarrow{\mathbb{1}}]}$. Hence $\tau$ can be iterated in a natural way.

Definition 7.2. The $\mathbb{Z}^{d}$ substitution system associated with a substitution rule $\tau$ is the set $\Omega$ of all $\omega \in \mathcal{A}^{\mathbb{Z}^{d}}$ so that every subword of $\omega$ is a subword of $\tau^{k}(a)$ for some $a \in \mathcal{A}$ and some $k \in \mathbb{N}_{0}$.

In [23], it was shown that for any $\mathbb{Z}^{d}$ substitution system $\Omega$ there exists an SFT $\Sigma$ and a one-block factor map $\varsigma$ from $\Sigma$ onto $\Omega$. If, in addition, the substitution system has a property called unique derivation, then this map can in fact be made into a measure-theoretic isomorphism, though a surjection will suffice for our purposes. Consider the $\mathbb{Z}^{d-1}$ substitution system $\Omega_{1}$ defined by the following substitution rule $\tau_{1}:\{0,1\} \rightarrow\{0,1\}^{[\overrightarrow{\mathbb{1}}, 9 \overrightarrow{\mathbb{1}}]}$ (in the picture, the $\vec{e}_{1}$-direction is horizontal):

$$
\begin{array}{lllllllllll}
0 & 0 & 0 & 0 & 0 & 0 & 0 & 0 & 0 \\
0 & 0 & 0 & 0 & 0 & 0 & 0 & 0 & 0 \\
0 & 0 & 0 & 0 & 0 & 0 & 0 & 0 & 0 \\
0 & 0 & 0 & 0 & 0 & 0 & 0 & 0 & 0 \\
0 & 0 & 0 & 0 & 0 & 0 & 0 & 0 & 0 \\
0 & 0 & 0 & 0 & 0 & 0 & 0 & 0 & 0 \\
0 & 0 & 0 & 0 & 0 & 0 & 0 & 0 & 0 \\
0 & 0 & 0 & 0 & 0 & 0 & 0 & 0 & 0 \\
0 & 0 & 0 & 0 & 0 & 0 & 0 & 0 & 0
\end{array}
$$$$
\begin{array}{llllllllll}
1 & 0 & 0 & 0 & 0 & 0 & 0 & 0 & 1 \\
1 & 0 & 0 & 0 & 0 & 0 & 0 & 0 & 1 \\
1 & 0 & 0 & 0 & 0 & 0 & 0 & 0 & 1 \\
1 & 0 & 0 & 0 & 0 & 0 & 0 & 0 & 1 \\
1 & 0 & 0 & 0 & 0 & 0 & 0 & 0 & 1 \\
1 & 0 & 0 & 0 & 0 & 0 & 0 & 0 & 1 \\
1 & 0 & 0 & 0 & 0 & 0 & 0 & 0 & 1 \\
1 & 0 & 0 & 0 & 0 & 0 & 0 & 0 & 1 \\
1 & 0 & 0 & 0 & 0 & 0 & 0 & 0 & 1
\end{array}
$$

The shown substitution rule is for $d-1=2$; for $d-1>2$ it is extended to the remaining directions by concatenating copies of the image-patterns in the picture above. Explicitly: $\tau_{1}(0):=0^{[\overrightarrow{\mathbb{1}}, 9 \overrightarrow{\mathbb{1}}]}$, and $\tau_{1}(1)$ is defined by $\left(\tau_{1}(1)\right)_{\vec{\jmath}}:=0$ if $\vec{\jmath}_{1} \in\{2,3, \ldots, 8\}$ and $\left(\tau_{1}(1)\right)_{\vec{\jmath}}:=1$ if $\vec{\jmath}_{1} \in\{1,9\}$ (for $\vec{\jmath} \in[\overrightarrow{\mathbb{1}}, 9 \overrightarrow{\mathbb{1}}]$ ).

Now by Mozes' theorem, choose a $\mathbb{Z}^{d-1}$ SFT $\Sigma_{1}$ which surjectively factors onto $\Omega_{1}$ via a one-block factor map $\varsigma_{1}$. Create $\mathbb{Z}^{d-1}$ SFTs $\Sigma_{k}$ for $2 \leq k \leq d-1$ in the same way; i.e. $\Sigma_{k}$ factors onto $\Omega_{k}$ via $\varsigma_{k}$, where $\Omega_{k}$ denotes the substitution system obtained from the rule $\tau_{k}(0):=0^{[\overrightarrow{\mathbb{1}}, 9 \overrightarrow{\mathbb{1}}]}$, and $\left(\tau_{k}(1)\right)_{\vec{\jmath}}:=0$ if $\vec{\jmath}_{k} \in\{2,3, \ldots, 8\}$ and $\left(\tau_{k}(1)\right)_{\vec{\jmath}}:=1$ if $\vec{\jmath}_{k} \in\{1,9\}$. Next, define the $\mathbb{Z}^{d-1}$ SFT $\Sigma:=\Sigma_{1} \times \Sigma_{2} \times \ldots \times \Sigma_{d-1}$ whose alphabet we denote by $\mathcal{A}_{\Sigma}$, as well as the sofic shift $\Lambda \subsetneq\{0,1\}^{\mathbb{Z}^{d-1}}$ obtained by applying the one-block factor map

$$
\eta: \Sigma \rightarrow \Lambda,\left(s_{1}, s_{2}, \ldots, s_{d-1}\right) \mapsto\left(\max \left\{\left(\varsigma_{k}\left(s_{k}\right)\right)_{\vec{\jmath}} \mid 1 \leq k \leq d-1\right\}\right)_{\vec{\jmath} \in \mathbb{Z}^{d-1}}
$$

which sends a symbol $\left(s_{1}, s_{2}, \ldots, s_{d-1}\right)_{\vec{\jmath}} \in \mathcal{A}_{\Sigma}$ from the alphabet of $\Sigma$ to 0 if $\left(\varsigma_{k}\left(s_{k}\right)\right)_{\vec{\jmath}}=0$ for all $1 \leq k \leq d-1$ and to 1 in any other case. $\Lambda$ then consists of $0 / 1$-configurations on $\mathbb{Z}^{d-1}$ with a grid structure of sparse $\mathbb{Z}^{d-2}$-hyperplanes of $1 \mathrm{~s}$ in each cardinal direction.

Claim 7.3. The number of 1 s within a solid block $B:=[\overrightarrow{\mathbb{1}}, n \overrightarrow{\mathbb{1}}] \subsetneq \mathbb{Z}^{d-1}$ in points of $\Lambda$ is bounded by $n^{(d-2)+.32}$ for large $n \in \mathbb{N}$. In particular, the frequency of 1s in any point of $\Lambda$ is zero, and thus every point in $\Lambda$ contains arbitrarily large $\mathbb{Z}^{d-1}$-hypercubes of $0 \mathrm{~s}$.

Proof. The sparsely appearing $\mathbb{Z}^{d-2}$-hyperplanes of 1 s along each $\vec{e}_{k}$-direction $(1 \leq$ $k \leq d-1)$ in points of $\Lambda$ stem from the substitution rule $\tau_{k}$. As $\tau_{k}(0)=0^{[\overrightarrow{\mathbb{1}}, 9 \overrightarrow{\mathbb{1}}]}$ an upper bound to the number of $\mathbb{Z}^{d-2}$-hyperplanes being perpendicular to $\vec{e}_{k}$ and 
carrying symbols 1 in a legal $\Lambda$-pattern of shape $B$ is achieved by applying the substitution rule $\left\lceil\log _{9} n\right\rceil$ times to the symbol 1 . In every iteration the number of $\mathbb{Z}^{d-2}$-hyperplanes of $1 \mathrm{~s}$ doubles and the total number of $1 \mathrm{~s}$ is multiplied by a factor $2 \cdot 9^{(d-2)}$. Simply adding the numbers produced in all the $d-1$ directions, we slightly overestimate the total number of 1 s, getting a bound

$$
\text { \# 1s in any } \begin{aligned}
P \in \mathcal{L}_{B}(\Lambda) & \leq(d-1) \cdot\left(2 \cdot 9^{(d-2)}\right)^{\left\lceil\log _{9} n\right\rceil} \\
& \leq(d-1) \cdot 2 \cdot 2^{\log _{9} n} \cdot 9^{(d-2)} \cdot n^{(d-2)}=C \cdot n^{(d-2)+\frac{\log 2}{\log 9}}
\end{aligned}
$$

with a constant $C:=2 \cdot 9^{(d-2)} \cdot(d-1)$. As $\frac{\log 2}{\log 9}<.3155$ we established the first part of our claim (increasing the exponent to $(d-2)+.32$ we get rid of the constant for large enough values of $n$ ).

Moreover, this bound shows that the frequency of $1 \mathrm{~s}$

$$
f_{(1)} \leq \lim _{n \rightarrow \infty} \frac{C \cdot n^{(d-2)+\frac{\log 2}{\log 9}}}{n^{(d-1)}}=C \cdot \lim _{n \rightarrow \infty} n^{\frac{\log 2}{\log 9}-1}=0
$$

is zero in every point of $\Lambda$, and thus in particular there can be no bound on the size of hypercubes of 0 s seen in each single point in $\Lambda$.

For each $1 \leq i \leq d$ let $\widetilde{\Sigma}_{i}:=\left\{\widetilde{s} \in \mathcal{A}_{\Sigma} \mathbb{Z}^{d}|\forall n \in \mathbb{Z}: \widetilde{s}|_{\left\{\vec{\jmath} \in \mathbb{Z}^{d} \mid \vec{\jmath}_{i}=n\right\}} \in \Sigma\right\}$ be the $\mathbb{Z}^{d}$ SFT obtained as the full $\mathbb{Z}$ extension of $\Sigma$ along direction $\vec{e}_{i}$. Define the $\mathbb{Z}^{d}$ SFT $\widetilde{W}_{i}$ constructed just as $W_{i}$, with the following changes: the alphabet of $\widetilde{W}_{i}$ is $\widetilde{\mathcal{A}}_{i} \cup \widetilde{\mathcal{A}}_{i}^{\prime}$, where $\widetilde{\mathcal{A}}_{i}:=\mathcal{A}_{i} \times \mathcal{A}_{\Sigma}$ and each element of $\widetilde{\mathcal{A}}_{i}^{\prime}$ is again a vertical line segment, this time labeled with a symbol of $\widetilde{\mathcal{A}}_{i}$. Points in $\widetilde{W}_{i}$ arise from elements in the SFT $V_{i} \times \widetilde{\Sigma}_{i}$ as points in $W_{i}$ arose from elements in $V_{i}$, with only one exception: in addition to the restrictions on adjacencies described for $W_{i}$ above, we introduce an extra rule allowing ascensions only at a sparse set of coordinates governed by the superimposed points from $\widetilde{\Sigma}_{i}$. More explicitly, for any $\vec{\imath} \in \mathbb{Z}^{d}$ and for any $1 \leq k \neq i \leq d$, if a symbol $\widetilde{a}=(a, s) \in \widetilde{\mathcal{A}}_{i}$ appears at $\vec{\imath}$, then a symbol $\widetilde{b}=(b, t) \in \widetilde{\mathcal{A}}_{i}$ is allowed to appear at $\vec{\imath}+\vec{e}_{k}+\vec{e}_{i}$ only when $\eta(t)=1$; otherwise, $\widetilde{b}$ has to appear at coordinate $\vec{\imath}+\vec{e}_{k}$. Claim 7.3 then shows that on a large scale ascensions of hyperplanes happen very rarely. Finally define the sofic shift $\widetilde{U}_{i}$ (a subsystem of $\left.U_{i}\right)$ as the image of $\widetilde{W}_{i}$ under the one-block map given by the rule $\widetilde{a}=(a, s) \mapsto \gamma_{i}(a)$ for all $\widetilde{a}=(a, s) \in \widetilde{\mathcal{A}}_{i}$ and $\widetilde{a}^{\prime} \mapsto 0$ for all vertical line segments $\widetilde{a}^{\prime} \in \widetilde{\mathcal{A}}_{i}^{\prime}$. Denote this one-block map by $\widetilde{\varpi}: \widetilde{W}_{i} \rightarrow \widetilde{U}_{i}$. A gently ascending hyperplane of a point in $\widetilde{U}_{i}$ is defined with respect to $\widetilde{\mathcal{A}}_{i}$ in the same way as a gently ascending hyperplane of a point in $U_{i}$ is defined with respect to $\mathcal{A}_{i}$. If $\widetilde{\varpi}(w)=u$, then a gently ascending hyperplane in $w \in \widetilde{W}_{i}$ is simply the preimage of a gently ascending hyperplane $H$ in $u \in \widetilde{U}_{i}$, and on a large scale $H$ has rare ascensions.

Next we have to prove some elementary properties of the sofic shifts $\widetilde{U}_{i}$ that will be used later.

Claim 7.4. The topological entropy of $\widetilde{U}_{i}(1 \leq i \leq d)$ is zero.

Proof. As all of the shifts $\widetilde{U}_{i}$ are constructed in the same way, it is enough to show that $h\left(\widetilde{U}_{1}\right)=0$. In order to get a sufficient (upper) bound on the entropy of $\widetilde{U}_{1}$, we do a very coarse estimate on the number of legal patterns in $\widetilde{U}_{1}$ on some large solid block $B_{n}:=[\overrightarrow{\mathbb{1}}, n \overrightarrow{\mathbb{1}}] \subsetneq \mathbb{Z}^{d}(n \in \mathbb{N})$. First note that a gently ascending hyperplane of 
1s which intersects $B_{n}$ must intersect the set $\left\{\overrightarrow{\mathbb{1}}+j \vec{e}_{1} \mid-d(n-1)<j<n\right\}$ at a single point. For $u \in \widetilde{U}_{1}$, define $J_{n}(u)$ as the set of $j \in \mathbb{Z}$ such that $-d(n-1)<j<n$ and $\overrightarrow{\mathbb{1}}+j \vec{e}_{1}$ is part of some gently ascending hyperplane of $1 \mathrm{~s}$ in $u$. Trivially, the number $\left|\left\{J_{n}(u) \mid u \in \widetilde{U}_{1}\right\}\right|$ is bounded above by $2^{n(d+1)}$. It is not hard to see that $\left.u\right|_{B_{n}}$ is uniquely determined - use the fact that $\widetilde{U}_{1}$ has alphabet $\{0,1\}$ - by the set $J_{n}(u)$, together with the sets of coordinates within each hyperplane at which ascensions actually occur inside $B_{n}$. The latter have to be subsets of the coordinates containing the symbol 1 in the corresponding superimposed $\Lambda$-point intersected with $B_{n}$, and their cardinality is bounded by $n^{(d-2)+.32}(17.3)$ for large $n$. Hence we get an upper bound on the number of valid $\widetilde{U}_{1}$-patterns on $B_{n}=[\overrightarrow{\mathbb{1}}, n \overrightarrow{\mathbb{1}}]$ with $n$ large enough:

$$
\left|\mathcal{L}_{B_{n}}\left(\widetilde{U}_{1}\right)\right| \leq 2^{n(d+1)} \cdot\left(\sum_{m=0}^{\left\lfloor n^{(d-1.68)}\right\rfloor}\left(\begin{array}{c}
n_{m}^{(d-1)} \\
m
\end{array}\right)\right)^{n(d+1)} .
$$

For $n$ large enough $n^{(d-1.68)} \ll \frac{1}{2} n^{(d-1)}$, so the binomial coefficients grow with $m$ and the sum can be bounded from above by taking the trivial approximation

$$
\begin{aligned}
\left|\mathcal{L}_{B_{n}}\left(\widetilde{U}_{1}\right)\right| & \leq 2^{n(d+1)} \cdot\left(n^{(d-1.68)}+1\right)^{n(d+1)} \cdot\left(\begin{array}{c}
n^{(d-1)} \\
\left\lfloor n^{(d-1.68)}\right\rfloor
\end{array}\right)^{n(d+1)} \\
& \leq\left(2 \cdot n^{(d-1.68)}+2\right)^{n(d+1)} \cdot\left(n^{(d-1)}\right)^{n^{(d-1.68)} \cdot n(d+1)} .
\end{aligned}
$$

Therefore the entropy of $\widetilde{U}_{1}$ turns out to be zero, as claimed above, by the following estimate:

$$
\begin{aligned}
0 \leq h\left(\widetilde{U}_{1}\right) & =\lim _{n \rightarrow \infty} \frac{\log \left|\mathcal{L}_{B_{n}}\left(\widetilde{U}_{1}\right)\right|}{n^{d}} \\
& \leq \lim _{n \rightarrow \infty} \frac{\log \left(\left(2 \cdot n^{(d-1.68)}+2\right)^{n(d+1)} \cdot n^{\left(d^{2}-1\right) \cdot n^{(d-0.68)}}\right)}{n^{d}}=0 .
\end{aligned}
$$

Claim 7.5. Every $\mathbb{Z}^{d}$ shift $\widetilde{U}_{i}(1 \leq i \leq d)$ as defined above is topologically mixing.

Proof. We can show that $\widetilde{U}_{i}$ is topologically mixing in much the same way as was done for the ribbon shift in 24]. Consider any two congruent solid blocks $B_{1}, B_{2} \subsetneq \mathbb{Z}^{d}$ spaced at a large distance $D_{B_{1}, B_{2}} \in \mathbb{N}$ apart and legal $\widetilde{U}_{i}$-patterns $P_{1} \in \mathcal{L}_{B_{1}}\left(\widetilde{U}_{i}\right)$ on $B_{1}$ and $P_{2} \in \mathcal{L}_{B_{2}}\left(\widetilde{U}_{i}\right)$ on $B_{2}$. (The definition of topological mixing uses arbitrary finite subsets, however it is easy to see that checking it for congruent solid blocks suffices.) Without loss of generality we may assume that in our patterns $P_{j}(j=1,2)$ there are no symbols 1 along the top or bottom of the solid blocks $B_{j}$. If this were the case, just use the fact that $P_{j}$ is a legal word in $\widetilde{U}_{i}$, say $P_{j}=\left.\widetilde{u}^{(j)}\right|_{B_{j}}$ for some $\widetilde{u}^{(j)} \in \widetilde{U}_{i}$, to extend it upwards and downwards to a pattern $P_{j}^{\prime}:=\left.\widetilde{u}^{(j)}\right|_{B_{j}^{\prime}}$ on a solid block $B_{j}^{\prime} \supseteq B_{j}$ in which every gently ascending hyperplane intersecting the original $P_{j}$ does not intersect the top or bottom of $B_{j}^{\prime}$. Then, replace all symbols in $P_{j}^{\prime}$ above the topmost hyperplane which intersects $P_{j}$ and below the bottommost hyperplane which intersects $P_{j}$ with $0 \mathrm{~s}$. This results in a pattern $P_{j}^{\prime \prime}$ (still on $B_{j}^{\prime}$ ) which is legal in $\widetilde{U}_{i}$ (since thickening layers of 0 s is always possible), which contains $P_{j}$ as a subpattern, and which has no hyperplanes of 1 s intersecting its top and bottom. As the slope of our gently ascending hyperplanes is at most 1 in cardinal directions, the required vertical extension of the pattern $P_{j}$ 
to a pattern $P_{j}^{\prime \prime}$ is linear in the size of $B_{j}$, and by adding some extra hyperplanes of 0s on top of the smaller one of the two patterns $P_{j}^{\prime \prime}$ if necessary, we reestablish our assumption that both patterns have a congruent shape. For notational simplicity from now on we will drop the 's, so talking about $P_{j}$ on $B_{j}$ we actually refer to the enlarged patterns constructed above.

\begin{tabular}{|c|c|c|c|c|c|c|c|c|c|c|c|c|}
\hline & \begin{tabular}{|l|l|}
0 & 0 \\
\end{tabular} & 1 & 10 & 0 & 0 & \begin{tabular}{l|l|l|}
1 & 0 & 0 \\
\end{tabular} & \begin{tabular}{|l|l|l|}
0 & 0 & 0 \\
\end{tabular} & 0 & & \begin{tabular}{l|l|l|}
0 & 0 \\
\end{tabular} & & $\begin{array}{lll}0 & 0 \\
\end{array}$ \\
\hline & \begin{tabular}{|l|l|}
1 & 1 \\
\end{tabular} & 00 & \begin{tabular}{l|l|l}
0 & 0 \\
\end{tabular} & 1 & 11 & \begin{tabular}{l|l|l|}
0 & 0 & 1 \\
\end{tabular} & \begin{tabular}{|l|l|l|}
0 & 0 & 0 \\
\end{tabular} & 0 & 0 & \begin{tabular}{l|l|l}
0 & 0 \\
\end{tabular} & $\underline{0}$ & 0 \\
\hline & \begin{tabular}{|l|l|}
0 & 0 \\
\end{tabular} & $0 \mid 1$ & & 0 & 0 & \begin{tabular}{l|l|l}
0 & 1 & 0 \\
\end{tabular} & \begin{tabular}{|l|l|l|}
0 & 0 & 0 \\
\end{tabular} & 0 & 0 & \begin{tabular}{l|l|}
0 & 0 \\
\end{tabular} & & 10 \\
\hline & \begin{tabular}{|l|l|}
0 & 1 \\
\end{tabular} & 10 & $\begin{array}{lll}0 & 0 \\
\end{array}$ & & 1 & \begin{tabular}{l|l|l|}
1 & 0 & 0 \\
\end{tabular} & \begin{tabular}{|l|l|l|}
0 & 0 & 0 \\
\end{tabular} & 0 & & \begin{tabular}{l|l|l|l|}
1 & 1 & \\
\end{tabular} & & \begin{tabular}{l|l}
0 & 0 \\
\end{tabular} \\
\hline & \begin{tabular}{|l|l|}
1 & 0 \\
\end{tabular} & 00 & 0 & 0 & 0 & \begin{tabular}{l|l|l|}
0 & 0 & 1 \\
\end{tabular} & \begin{tabular}{|l|l|l|}
0 & 0 & 0 \\
\end{tabular} & 0 & & \begin{tabular}{l|l|l}
0 & 0 \\
\end{tabular} & & \\
\hline \begin{tabular}{|l|l|l|l|l|l|l|l|l|l|}
0 & 0 & 0 & 1 & 0 & 0 & 0 & 0 & 1 & 0 \\
\end{tabular} & \begin{tabular}{|l|l|}
0 & 0 \\
\end{tabular} & 0[ & 10 & 0 & $\overline{0}$ & \begin{tabular}{ll|l}
0 & 1 & 0 \\
\end{tabular} & \begin{tabular}{l|l|l|l|l|l|l|l|} 
\\
\end{tabular} & 1 & 0 & \begin{tabular}{ll|}
0 & 0 \\
\end{tabular} & 0 & $\begin{array}{lll}1 & 0 \\
\end{array}$ \\
\hline \begin{tabular}{|l|l|l|l|l|l|l|l|l|l|}
0 & 1 & 1 & 0 & 0 & 0 & 1 & 1 & 0 & 0 \\
\end{tabular} & 01 & 10 & \begin{tabular}{l|l|}
0 & 0 \\
\end{tabular} & 0 & 1 & \begin{tabular}{l|l|l|}
1 & 0 & 0 \\
\end{tabular} & \begin{tabular}{l|l|l}
0 & 1 & 1 \\
\end{tabular} & 10 & 0 & \begin{tabular}{l|l|}
0 & 1 \\
\end{tabular} & 1 & \begin{tabular}{l|l|l}
0 & 0 \\
\end{tabular} \\
\hline \begin{tabular}{|l|l|l|l|l|l|l|l|l|l|}
1 & 0 & 0 & 0 & 0 & 1 & 0 & 0 & 0 & 0 \\
\end{tabular} & 10 & 00 & \begin{tabular}{|l|l|}
0 & 0 \\
\end{tabular} & 1 & 0 & \begin{tabular}{l|l|l|}
0 & 0 & 0 \\
\end{tabular} & \begin{tabular}{|l|l|l|}
1 & 0 & 0 \\
\end{tabular} & 0 & 0 & \begin{tabular}{l|l|l|}
1 & 0 & \\
\end{tabular} & $\underline{0}$ & $\begin{array}{lll}0 & 0 . \\
\end{array}$ \\
\hline \begin{tabular}{|l|l|l|l|l|l|l|l|l|l|}
0 & 0 & 0 & 1 & 1 & 0 & 0 & 0 & 0 & 1 \\
\end{tabular} & \begin{tabular}{|l|l|}
0 & 0 \\
\end{tabular} & $0 \mid 1$ & \begin{tabular}{l|l|}
1 & 1 \\
\end{tabular} & 0 & 0 & \begin{tabular}{l|l|l|}
0 & 0 & 1 \\
\end{tabular} & \begin{tabular}{|l|l|l|}
0 & 0 & 0 \\
\end{tabular} & 1 & 1 & \begin{tabular}{l|l|l}
0 & 0 \\
\end{tabular} & $\underline{0}$ & \\
\hline \begin{tabular}{|l|l|l|l|l|l|l|l|l|}
0 & 0 & 1 & 0 & 0 & 0 & 0 & 0 & 1 \\
\end{tabular} & \begin{tabular}{|l|l|}
0 & 0 \\
\end{tabular} & 10 & \begin{tabular}{l|l}
0 & 0 \\
\end{tabular} & 0 & 0 & \begin{tabular}{l|l|l|}
0 & 1 & 0 \\
\end{tabular} & \begin{tabular}{|l|l|l|}
0 & 0 & 1 \\
\end{tabular} & 0 & 0 & \begin{tabular}{l|l|}
0 & 0 \\
\end{tabular} & 0 & 10 \\
\hline \begin{tabular}{|l|l|l|l|l|l|l|l|l|l|}
0 & 1 & 0 & 0 & 0 & 0 & 0 & 1 & 0 & 0 \\
\end{tabular} & \begin{tabular}{|l|l|}
0 & 1 \\
\end{tabular} & 010 & \begin{tabular}{l|l}
0 & 0 \\
\end{tabular} & 10 & 0 & 100 & \begin{tabular}{|l|l|l}
0 & 1 & 0 \\
\end{tabular} & 0 & 0 & \begin{tabular}{ll|l}
0 & 0 \\
\end{tabular} & & 0 \\
\hline & \begin{tabular}{|l|l|}
1 & 0 \\
\end{tabular} & 00 & $\begin{array}{ll}0 & 0 \\
\end{array}$ & & 1 & \begin{tabular}{l|ll}
0 & 0 & 0 \\
\end{tabular} & \begin{tabular}{|l|l|l|}
1 & 0 & 0 \\
\end{tabular} & 0 & 0 & 11 & & \begin{tabular}{|l|l|}
0 & 0 \\
\end{tabular} \\
\hline & \begin{tabular}{|l|l|l}
0 & 0 \\
\end{tabular} & 0 & 111 & 0 & 0 & \begin{tabular}{l|l|l|}
0 & 1 & 1 \\
\end{tabular} & \begin{tabular}{|l|l|l}
0 & 0 & 0 \\
\end{tabular} & 1 & 1 & \begin{tabular}{l|l|l|}
0 & 0 \\
\end{tabular} & & \begin{tabular}{l|l}
0 & 0 \\
\end{tabular} \\
\hline & \begin{tabular}{|l|l|}
0 & 0 \\
\end{tabular} & 10 & \begin{tabular}{l|l|}
0 & 0 \\
\end{tabular} & 0 & 0 & \begin{tabular}{l|l|l}
1 & 0 & 0 \\
\end{tabular} & \begin{tabular}{|l|l|l|l|}
0 & 0 & 1 \\
\end{tabular} & 10 & 0 & \begin{tabular}{l|l|l|}
0 & 0 \\
\end{tabular} & & \begin{tabular}{l|l}
0 & 0 \\
\end{tabular} \\
\hline & \begin{tabular}{|l|l|}
0 & 1 \\
\end{tabular} & 00 & \begin{tabular}{l|l|}
0 & 0 \\
\end{tabular} & 0 & 1 & \begin{tabular}{|l|l|l}
0 & 0 & 0 \\
\end{tabular} & \begin{tabular}{|l|lll}
0 & 0 \\
\end{tabular} & 5 & 0 & \begin{tabular}{l|l|}
0 & 0 \\
\end{tabular} & 0 & \begin{tabular}{l|l}
0 & 0 \\
\end{tabular} \\
\hline & \begin{tabular}{|l|l|}
1 & 0 \\
\end{tabular} & 00 & \begin{tabular}{l|l|}
0 & 1 \\
\end{tabular} & 1 & 0 & \begin{tabular}{l|l|l}
0 & 0 & 1 \\
\end{tabular} & \begin{tabular}{|l|l}
1 & 0 \\
\end{tabular} & 0 & 0 & \begin{tabular}{|l|l|}
0 & 0 \\
\end{tabular} & 0 & \begin{tabular}{l|l}
0 & 0 \\
\end{tabular} \\
\hline & \begin{tabular}{|l|l|}
0 & 0 \\
\end{tabular} & \begin{tabular}{|l|l}
0 & 1 \\
\end{tabular} & $\begin{array}{l}1 \\
1\end{array}$ & 0 & 0 & \begin{tabular}{l|l|l}
0 & 1 & 0 \\
\end{tabular} & \begin{tabular}{|l|l|l|l|}
0 & 0 & 0 \\
\end{tabular} & & 0 & \begin{tabular}{l|l|l|}
0 & 0 \\
\end{tabular} & & \begin{tabular}{l|l}
0 & 0 \\
\end{tabular} \\
\hline
\end{tabular}

FIgure 4. Extending a pattern $P_{j}$ on the solid block $B_{j}$ (on the left) via a pattern $P_{j}^{\prime}$ on $B_{j}^{\prime}$ (in the middle) to a pattern $P_{j}^{\prime \prime}$ also on $B_{j}^{\prime}$ (on the right).

After this preprocessing there are two cases: either $B_{1}$ and $B_{2}$ are separated by a large distance in the $\vec{e}_{i}$-direction, or they have large separation in an $\vec{e}_{k}$-direction for some $1 \leq k \neq i \leq d$. If they have large separation in the $\vec{e}_{i}$-direction, then the fact that the patterns $P_{1}$ and $P_{2}$ can be put together to form a valid point in $\widetilde{U}_{i}$ comes from the mixing of $Z$ (condition 1 of Lemma 5.1). To create a $\widetilde{U}_{i^{-}}$ configuration on $\mathbb{Z}^{d}$ containing $P_{1}$ on $B_{1}$ and $P_{2}$ on $B_{2}$, simply first extend all gently ascending (finite) regions of 1 s inside $P_{1}$ and $P_{2}$ to ascending hyperplanes of 1 s with no additional ascensions (apart from the ones forced by the homogeneity condition) outside $B_{1}$ and $B_{2}$. Fill the gaps between those hyperplanes with 0 s and add a layer of 0 s just above and below the extended patterns. Then use the mixing of $Z$ to interpolate between those infinite configurations, putting in non-ascending hyperplanes of $1 \mathrm{~s}$ and 0 s surrounded vertically by layers of 0 s according to a point in $Z$ that contains subwords realizing both patterns $P_{1}$ and $P_{2}$. As long as the $\vec{e}_{i}$-separation is large enough, this can always be done.

Suppose now that $B_{1}$ and $B_{2}$ have large separation in some direction $\vec{e}_{k}$ with $k \neq i$. In this case we exploit the possibility of letting hyperplanes of 1 s ascend in order to interpolate between $P_{1}$ and $P_{2}$. Roughly speaking, we again extend the gently ascending (finite) regions of 1 s inside $P_{1}$ and $P_{2}$ to ascending hyperplanes, but outside $B_{1}$ and $B_{2}$ we allow only ascensions in the $\vec{e}_{k}$-direction, apart from the ascensions forced by the homogeneity constraints. Then, interpolating between these two sets of hyperplanes in $\widetilde{U}_{i}$ may be done as in the first case, since due to the ascending and the sufficiently large initial $\vec{e}_{k}$-separation of $B_{1}$ and $B_{2}$, the two stacks of hyperplanes observed at the same $\vec{e}_{k}$-coordinate will already have large 
separation in the $\vec{e}_{i}$-direction. We have then shown that as long as $B_{1}$ and $B_{2}$ have large separation in some direction, $P_{1}$ and $P_{2}$ can be put together to form a valid point of $\widetilde{U}_{i}$, and so $\widetilde{U}_{i}$ is mixing.

We now proceed just as in Section [6] Take the product shift $\widetilde{U}_{1} \times \widetilde{U}_{2} \times \ldots \times \widetilde{U}_{d}$ and factor it onto the $\mathbb{Z}^{d}$ shift $\widetilde{S}^{\prime} \subsetneq\{0,1\}^{\mathbb{Z}^{d}}$ by the one-block factor map

$$
\widetilde{\vartheta}: \widetilde{U}_{1} \times \widetilde{U}_{2} \times \ldots \times \widetilde{U}_{d} \rightarrow \widetilde{S}^{\prime},\left(\widetilde{u}^{(1)}, \widetilde{u}^{(2)}, \ldots, \widetilde{u}^{(d)}\right) \mapsto\left(\widetilde{u}_{\vec{\imath}}^{(1)} \cdot \widetilde{u}_{\vec{\imath}}^{(2)} \cdot \ldots \cdot \widetilde{u}_{\vec{\imath}}^{(d)}\right)_{\vec{\imath} \in \mathbb{Z}^{d}} .
$$

As each $\widetilde{U}_{i}$ was a topologically mixing sofic shift, $\widetilde{S}^{\prime}$ is a mixing sofic as well. We also note that $h\left(\widetilde{S}^{\prime}\right)=0$; because $\widetilde{S}^{\prime}$ is a factor of $\widetilde{U}_{1} \times \widetilde{U}_{2} \times \ldots \times \widetilde{U}_{d}$, this follows from our Claim [7.4] Just as every point of $T_{i}$ contained syndetically appearing thick hyperplanes of 0 s, every point of $\widetilde{U}_{i}$ contains a set of arbitrarily thick gently ascending hyperplanes of $0 \mathrm{~s}$.

The next result shows that hyperplane ascensions in points of $\widetilde{U}_{i}$ are sufficiently slow so that we may recover the key feature of the gridlike structure of 0 s from $U_{i}$ : finite patterns must occur within block patterns which are labeled with $0 \mathrm{~s}$ in an arbitrarily large neighborhood of the boundary.

Claim 7.6. Suppose $\widetilde{s} \in \widetilde{S}^{\prime}$ and $l, m \in \mathbb{N}$. Then there exists a solid block $B:=$ $[\vec{v}, \vec{w}] \subsetneq \mathbb{Z}^{d}$, containing $[-(l+m) \overrightarrow{\mathbb{1}},(l+m) \overrightarrow{\mathbb{1}}]$, such that $\widetilde{s}_{\vec{\jmath}}=0$ whenever $\vec{\jmath} \in$ $[\vec{v}, \vec{w}] \backslash[\vec{v}+l \overrightarrow{\mathbb{1}}, \vec{w}-l \overrightarrow{\mathbb{1}}]$.

Proof. We start with a technical observation about the variation in thickness of layers of 0 s in points of $\widetilde{U}_{i}(1 \leq i \leq d)$. Suppose somewhere in a point $\widetilde{u} \in \widetilde{U}_{i}$ there appears a block of $n \in \mathbb{N}$ consecutive 0 s along the $\vec{e}_{i}$-direction. For convenience, assume this happens on the interval $[0, n-1] \vec{e}_{i}:=\left\{j \vec{e}_{i} \in \mathbb{Z}^{d} \mid 0 \leq j<n\right\} \subsetneq \mathbb{Z}^{d}$. We claim that for large $n, \widetilde{u}_{\vec{\jmath}}=0$ as well for any $\vec{\jmath} \in \mathbb{Z}^{d}$ with $\frac{n}{4} \leq \vec{\jmath}_{i}<\frac{3 n}{4}$ and $\|\vec{\jmath}\|_{\infty} \leq n^{3.1}+m+l$. In other words, within a distance of $n^{3.1}+m+l$ in all directions, hyperplanes do not ascend by more than $\frac{n}{4}$. This is not hard to see: consider the ascending hyperplanes of $1 \mathrm{~s}$ which bound our pattern of $0 \mathrm{~s}$ containing the interval $[0, n-1] \vec{e}_{i}$ from above and below. If those do not exist, i.e. the region of $0 \mathrm{~s}$ is unbounded from above/from below, the claim is trivially true. Along each direction $\vec{e}_{k}(k \neq i)$, these bordering hyperplanes may ascend only at coordinates where there is a $\mathbb{Z}^{d-2}$-hyperplane of 1 s perpendicular to $\vec{e}_{k}$ in the superimposed $\Lambda$-point. By earlier computations (see Claim 7.3 ) the number of those over a distance of $n^{3.1}+m+l$ is bounded by $\left(n^{3.1}+m+l\right)^{\frac{\log 2}{\log 9}}$. As the hyperplanes in $\widetilde{U}_{i}$ "climb up" just one coordinate in each ascension, after summing over all $\vec{e}_{k}$-directions, we can (over)estimate the total gain in height in direction $\vec{e}_{i}$ by $(d-1) \cdot\left(n^{3.1}+m+l\right)^{.32}<(d-1) \cdot n^{.993}<\frac{n}{4}$ for $n$ large enough.

Now consider any point $\widetilde{s} \in \widetilde{S}^{\prime}$ and some $\widetilde{\vartheta}$-preimage $\left(\widetilde{u}^{(1)}, \widetilde{u}^{(2)}, \ldots, \widetilde{u}^{(d)}\right) \in$ $\widetilde{\vartheta}^{-1}(\widetilde{s})$. The construction of $\widetilde{U}_{i}$ ensures that the point $\widetilde{u}^{(i)}$ contains the word $0^{n}$ somewhere in the interval $\left[m+l, n^{3.1}+m+l-1\right] \vec{e}_{i}$ and also somewhere in the interval $\left[-\left(n^{3.1}+m+l-1\right),-(m+l)\right] \vec{e}_{i}$. (Here $n^{3.1}$ appears, rather than the distance $n^{2.1}$ from the definition of $Z$, to address the fact that successive hyperplanes of 1 s are separated vertically with gap at most $n$.) We define the $i$ th coordinates of the vectors $\vec{v}, \vec{w}$ as follows. Using the technical result of the previous paragraph, we pick some interval $I_{i}=\left[\vec{w}_{i}-\frac{n}{2}, \vec{w}_{i}\right] \subseteq\left[m+l, n^{3.1}+m+l-1\right] \cap \mathbb{Z}$, such that $\widetilde{u}_{\vec{\jmath}}^{(i)}=0$ for any $\vec{\jmath} \in \mathbb{Z}^{d}$ with $\vec{\jmath}_{i} \in I_{i}$ and $\|\vec{\jmath}\|_{\infty} \leq n^{3.1}+m+l$. Similarily we pick an interval 
$\left[\vec{v}_{i}, \vec{v}_{i}+\frac{n}{2}\right]$ on the negative $\vec{e}_{i}$-axis. After applying $\widetilde{\vartheta}$, we see that the restriction of $\widetilde{s}$ to $[\vec{v}, \vec{w}]$ has 0 s at sites within distance $\frac{n}{2}$ of the boundary. We may require $\frac{n}{2}>l$, and this proves the claim.

If $z \in Z$, then for each $\widetilde{U}_{i}$ there is a point $\widetilde{u}^{(i)} \in \widetilde{U}_{i}$ such that $\widetilde{u}_{\vec{\jmath}}^{(i)}:=z_{n}$ when $\vec{\jmath}_{i}=2 n$ and $\widetilde{u}_{\vec{\jmath}}^{(i)}:=0$ otherwise. Since the symbol 1 occurs with positive frequency in some points of $Z$, it follows that 1 s appear with positive frequency in some points of $\widetilde{U}_{i}$ and therefore in some point of $\widetilde{S}^{\prime}$. Hence, just as in Section 6 we can split the symbol 1 into many distinct copies to create the $\mathbb{Z}^{d}$ sofic shift $\widetilde{S}$ of arbitrarily large entropy $h(\widetilde{S})>M$. Denote by $\widetilde{\kappa}: \widetilde{S} \rightarrow \widetilde{S}^{\prime}$ the 1-block factor map collapsing the different copies of the symbol 1 in $\widetilde{S}$ back onto the original. Since $\widetilde{S}^{\prime}$ was topologically mixing, it is clear that $\widetilde{S}$ is as well.

The rest of the proof of Theorem 4.1 for $d \geq 3$ now proceeds just as in the nonmixing case, with one exception. We do not claim that our mixing example $\widetilde{S}^{\prime}$ has a topological factor of completely positive entropy. (In fact, it has no such factor, but we will not give a proof.) Presumably this feature of the mixing example is only a limitation of our technique.

\section{The $\mathbb{Z}^{d}$ SFT EXAMPles For $d \geq 3$}

In this section we prove Theorem 4.2 in the case $d \geq 3$. We choose the required SFT $X$ to be the SFT cover of the sofic shift $S$ described in Theorem 4.1(7). We now verify the claims of Theorem 4.2

Proof. (11) This is instantly true as $X$ is a cover of $S$ and $h(S)>M$.

(2) Let $K$ be the SFT from part (7) of Theorem 4.1. If there is a minimal subsystem $W$ of $X$ which is not contained in $K$, then it is disjoint from $K$, and we have $\pi(W)$ a minimal subsystem of $S$ other than its fixed point 44.1(7)), contradicting part (2) of Theorem 4.1

(31), (41) Suppose $Y$ is non-trivial block gluing and $\phi: X \rightarrow Y$ is a factor map. Using Theorem B.2, we have $h(Y)>0$ (by non-triviality) and then $Y$ contains a subsystem $W$ disjoint from $\phi(K)$. Therefore $\phi^{-1}(W)$ is a subsystem of $X$ disjoint from $K$, which is impossible. As every minimal subsystem in $Y$ is contained in $\phi(K)$ and $h(\phi(K))=0$, we have $h\left(Y_{\mathrm{MIN}}\right)=0$.

(5) Because arbitrarily large hypercubes of 0 s occur in every point of $S$, and $K$ is the preimage of the fixed point $0^{\mathbb{Z}^{d}}$, it follows that arbitrarily large hypercubes of $K$-configurations occur in every point of $X$. Because $h(K)=0$, this claim (like Theorem 4.1(5)) now follows from Proposition 6.1.

(6) This follows because $S$ is an equal entropy factor of $X$ (4.1)(6)).

\section{A topologically MiXing, top.C.P.E. $\mathbb{Z}^{2}$ SFT eXAmple}

Turning our attention to two-dimensional shifts, we present a family of $\mathbb{Z}^{2}$ SFTs $\widetilde{X}_{k}$ which prove the case $d=2$ of Theorem 4.2 (with $X=\widetilde{X}_{k}$ of sufficiently large entropy).

These SFTs are in fact an example of Hochman from [12. We will begin by giving a brief description of the construction of those $\mathbb{Z}^{2}$ SFTs and discuss their properties pertinent to our argument. For a full examination of these systems, see $[12$. 
The construction: For any positive integer $k \in \mathbb{N}$, first a $\mathbb{Z}^{2}$ SFT $X_{k}$ is defined with alphabet $\mathcal{A}_{k}$ of $32+k$ symbols, which are most conveniently thought of as square tiles of unit length. Thirty-two of the symbols of $\mathcal{A}_{k}$ are defined by assigning to a tile one of four colors and one of eight types of arrows. For reasons which will soon become clear, the colors should be thought of as representing the four directions NW (northwest), NE (northeast), SW (southwest), and SE (southeast). The eight types of arrows are four straight arrows in the four cardinal directions (up, down, right, left) and four arrows turning ninety degrees clockwise. The remaining $k$ tiles are called "blanks" and are labeled with an associated integer between 1 and $k$ inclusive. The symbols of $\mathcal{A}_{k}$ appear in Figure 5

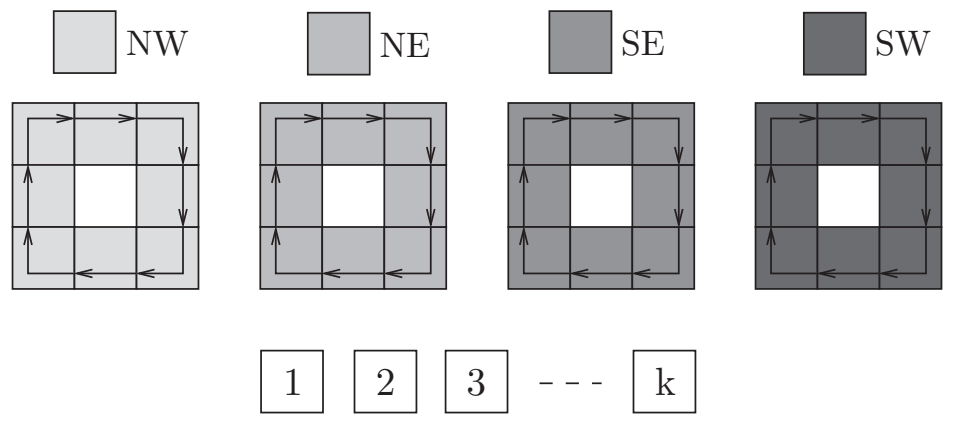

Figure 5 . The $32+k$ different symbols of $\mathcal{A}_{k}$.

The subshift $X_{k}$ is an SFT of order 2, meaning that there is a set of legal $2 \times 2$ patterns $\mathcal{L}_{\{0,1\}^{2}}\left(X_{k}\right) \subseteq \mathcal{A}_{k}{ }^{\{0,1\}^{2}}$, and $X_{k}$ is precisely the set of elements of $\mathcal{A}_{k} \mathbb{Z}^{2}$ in which every $2 \times 2$ subpattern is one of the elements in $\mathcal{L}_{\{0,1\}^{2}}\left(X_{k}\right)$. For the purposes of legal $2 \times 2$ patterns, all blanks are considered indistinguishable, and so from now on we refer only to blank tiles in general, with the understanding that the $k$ blank tiles are completely interchangeable in elements of $X_{k}$.

We inductively define valid patterns $P_{n}^{\theta} \in \mathcal{L}_{\left[\overrightarrow{1},\left(2^{n+1}+2^{n-1}-2\right) \overrightarrow{1}\right]}\left(X_{k}\right)$ for any $n \in \mathbb{N}$ and $\theta$ as one of the four colors $N W, N E, S E$, and $S W$ as follows (let us denote by $\mathcal{T}:=\{N W, N E, S E, S W\}$ the set of colors): for any $\theta \in \mathcal{T}, P_{1}^{\theta}$ is the $3 \times 3$ square with a blank tile in the center, surrounded by a clockwise circuit of arrows colored by $\theta$; for any $n \in \mathbb{N}$ and color $\theta \in \mathcal{T}, P_{n+1}^{\theta}$ is constructed by concatenating the four smaller patterns $P_{n}^{N W}, P_{n}^{N E}, P_{n}^{S E}$, and $P_{n}^{S W}$ into a square configuration of shape $\left[\overrightarrow{\mathbb{1}},\left(2\left(2^{n+1}+2^{n-1}-2\right)\right) \overrightarrow{\mathbb{1}}\right]$, with each of the four patterns $P_{n}^{\theta^{\prime}}$ in the corner corresponding to $\theta^{\prime} \in \mathcal{T}$, and then surrounding this square with a clockwise circuit of arrows, colored by $\theta$. For $n \in \mathbb{N}, \theta \in \mathcal{T}$, we call $P_{n}^{\theta}$ the level- $n$ square with $\theta$-colored boundary. As an example, the configuration appearing in Figure 6 is actually $P_{3}^{N W}$. The set $\mathcal{L}_{\{0,1\}^{2}}\left(X_{k}\right)$ of allowed $2 \times 2$ patterns in $X_{k}$ is defined to be the set of all $2 \times 2$ configurations which appear in $P_{3}^{\theta}$ for some $\theta \in \mathcal{T}$. It is verified in 12 that $P_{n}^{\theta} \in \mathcal{L}\left(X_{k}\right)$ for all $n \in \mathbb{N}, \theta \in \mathcal{T}$, and in fact we will see that in some sense most points in $X_{k}$ are built up from the patterns $P_{n}^{\theta}$. (We are still not concerning ourselves with the labeling of blank tiles, so each $P_{n}^{\theta}$ actually corresponds to many configurations in $\mathcal{L}(X)$ depending on how the blanks are labeled.) 


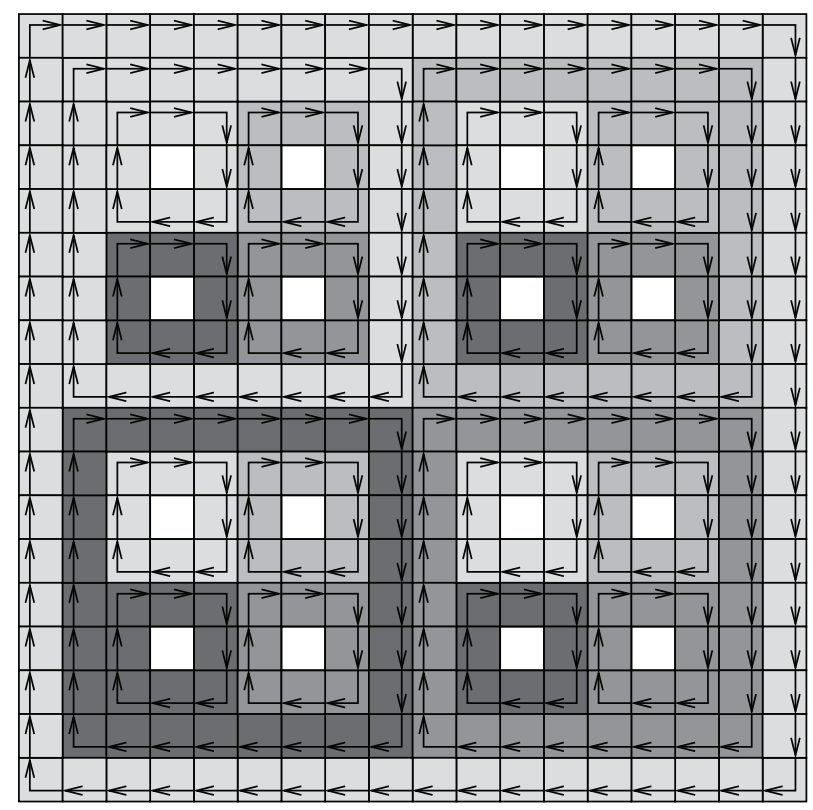

Figure 6. Part of a point of $X_{k}$.

For any $\omega=\left(\omega_{1}, \omega_{2}, \ldots\right) \in \mathcal{T}^{\mathbb{N}}$, we define $x_{\omega} \in X_{k}$ as follows: begin by defining $x_{\omega}$ on a $3 \times 3$ square centered at the origin, call this square $B_{1}:=[-\overrightarrow{\mathbb{1}}, \overrightarrow{\mathbb{1}}]$, so that $\left.x_{\omega}\right|_{B_{1}}:=P_{1}^{\omega_{1}}$. For any $n \geq 1$, assume that $x_{\omega}$ has already been defined on a square $B_{n} \subsetneq \mathbb{Z}^{2}$ which is a translate of $\left[\overrightarrow{\mathbb{1}},\left(2^{n+1}+2^{n-1}-2\right) \overrightarrow{\mathbb{1}}\right]$ so that $\left.x_{\omega}\right|_{B_{n}}=$ $P_{n}^{\omega_{n}}$. Since $P_{n}^{\omega_{n}}$ appears as a subpattern of $P_{n+1}^{\omega_{n+1}}$ exactly once, there is a unique way to extend the definition of $x_{\omega}$ to a square $B_{n+1}$ which is some translate of $\left[\overrightarrow{\mathbb{1}},\left(2^{(n+1)+1}+2^{(n+1)-1}-2\right) \overrightarrow{\mathbb{1}}\right]$, so that $\left.x_{\omega}\right|_{B_{n+1}}:=P_{n+1}^{\omega_{n+1}}$. In addition, as $P_{n+1}^{\omega_{n+1}}$ has a closed path of arrows on the boundary, it must be the case that $B_{n} \subseteq B_{n+1} \backslash \partial B_{n+1}$. It is then easily checked by induction that $[-n \overrightarrow{\mathbb{1}}, n \overrightarrow{\mathbb{1}}] \subseteq B_{n}$ for all $n \in \mathbb{N}$, and so $\bigcup_{n=1}^{\infty} B_{n}=\mathbb{Z}^{2}$; thus $x_{\omega}$ is eventually defined on all of $\mathbb{Z}^{2}$ in this way.

In [12, it is proven that every element $x \in X_{k}$ is of one of two types. If $x=\sigma^{\vec{r}}\left(x_{\omega}\right)$ for some $\vec{\imath} \in \mathbb{Z}^{2}$ and $\omega \in \mathcal{T}^{\mathbb{N}}$, then $x$ is transitive. Otherwise $x$ - being a boundary point of the orbit closure of some $x_{\omega}$ - is what Hochman calls an "exceptional point." Essentially there are four types of exceptional points, shown in Figure 7 without their coloring. Every exceptional point is of one of these four types, possibly rotated by a multiple of ninety degrees, and having the arrows colored in some way allowed in $X_{k}$.

For any subpattern of an exceptional point with shape $B:=[\overrightarrow{\mathbb{1}}, n \overrightarrow{\mathbb{1}}]$, knowing the arrows on the boundary of $B$ suffices to reconstruct the arrows of all interior tiles (there are no blank tiles in an exceptional point, and all arrows have to line up). If one knows in addition the colors of the boundary tiles, one can recover the colors in the interior. This is because, due to the finite type restrictions on $X_{k}$, any pair of arrows which join up head-to-tail must have the same color. This means that the 


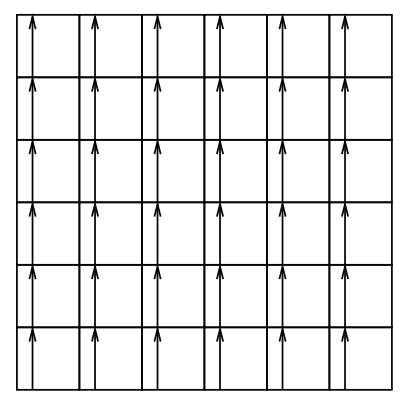

(a)

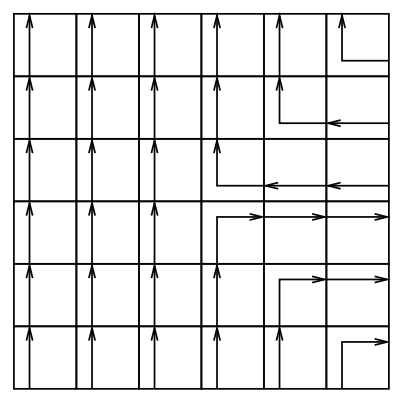

(c)

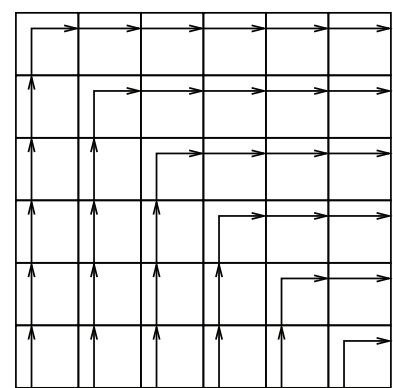

(b)

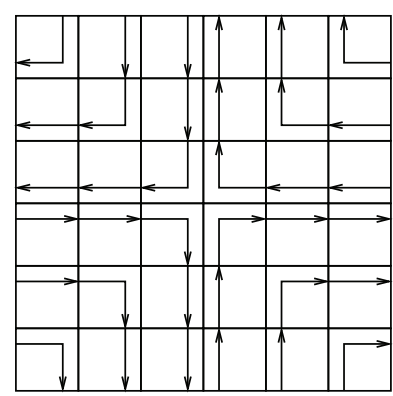

(d)

Figure 7. Exceptional points of $X_{k}$ (uncolored).

boundary of any subpattern of an exceptional point forces the configuration on its interior. So if we denote by $E \subsetneq X_{k}$ the subshift of $X_{k}$ consisting of all exceptional points, $\left|\mathcal{L}_{[\overrightarrow{\mathbb{1}}, n \overrightarrow{\mathbb{1}}]}(E)\right| \leq\left|\mathcal{A}_{k}\right|^{4 n-4}<(32+k)^{4 n}$, and so $h(E)=0$. (We may use the notation $E$ independent of $k$ since elements of $E$ contain no blanks, and therefore the subshifts of exceptional points in $X_{k}$ for any $k \in \mathbb{N}$ coincide.)

It is easily seen that no $X_{k}$ is topologically mixing, so the $X_{k}$ will not satisfy Theorem 4.2 (6). However, without much effort it is possible to adjust the defining rules of the SFTs $X_{k}$ in order to create similar $\mathbb{Z}^{2}$ shifts of finite type which are indeed topologically mixing. For this, we use another example of Hochman, which is very similar to $X_{k}$. In $X_{k}$, blank tiles were not permitted to be adjacent to each other. Define a new system $\widetilde{X}_{k}$ by allowing free combinations of blank tiles to appear in rectangular configurations of dimensions $1 \times 1,1 \times 2,2 \times 1$, and $2 \times 2$ only. All other adjacency conditions remain unchanged. Figure 8 shows a possible configuration in $\tilde{X}_{k}$.

The level- $n$ squares from $X_{k}$ correspond to a slightly more general class of level$n$ rectangles in $\widetilde{X}_{k}$. A level-1 rectangle of $\widetilde{X}_{k}$ is defined to be any rectangular configuration of blanks surrounded by a closed path of arrows (all with the same color). For $n>1$, a pattern with rectangular shape is a level- $n$ rectangle in $\widetilde{X}_{k}$ if the configuration on its boundary is a closed path of arrows with the same color and its interior is the union of four rectangular patterns, each of which is a level- $m$ rectangle for some $m<n$, and at least one of which is of level $n-1$. In fact, it is shown in Lemma 6.14 of 12 that the four component rectangular configurations must all be 


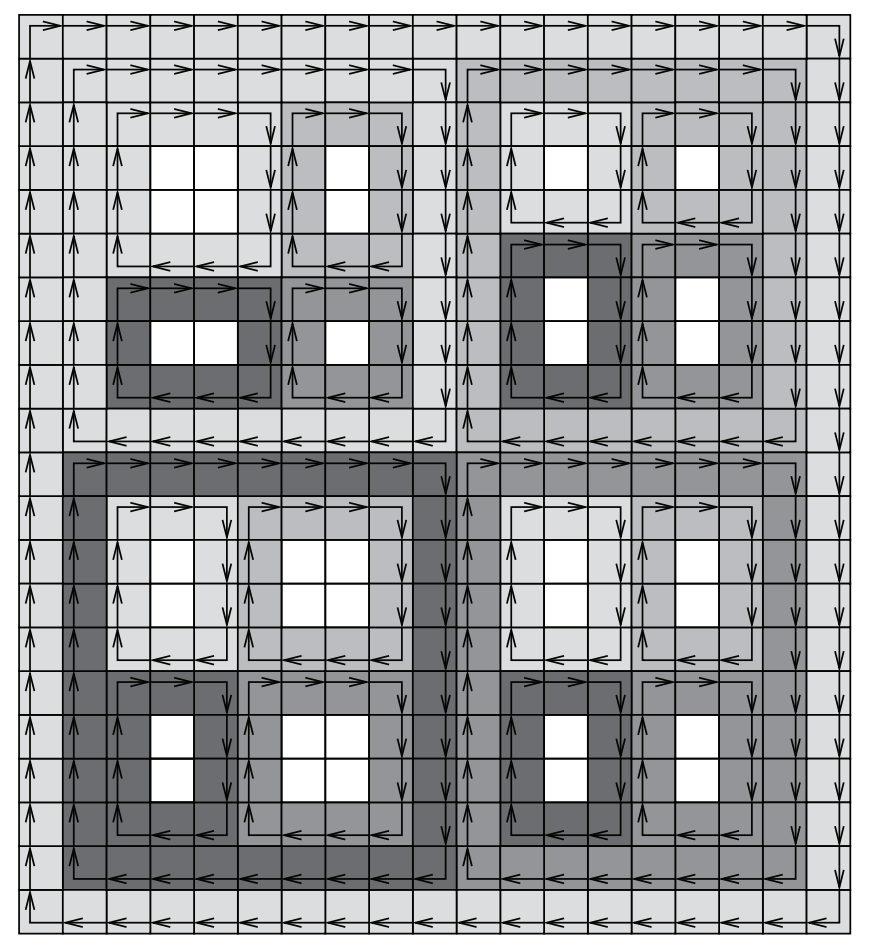

Figure 8. Part of a point of $\widetilde{X}_{k}$.

level- $(n-1)$ rectangles. The description of the transitive (non-exceptional) points of $\widetilde{X}_{k}$ is similar to that of $X_{k}$; Lemma 6.16 of 12 states that for any $\widetilde{x} \in \widetilde{X}_{k}$, either there exists a sequence of nested rectangles $R_{n} \subsetneq \mathbb{Z}^{2}(n \in \mathbb{N})$ with $\bigcup_{n=1}^{\infty} R_{n}=\mathbb{Z}^{2}$ where $\left.\widetilde{x}\right|_{R_{n}}$ is a level- $n$ rectangle for all $n \in \mathbb{N}$, or $\widetilde{x}$ is an exceptional point. (The set of exceptional points in $\widetilde{X}_{k}$ is actually the same as the set of exceptional points for $X_{k}$, again because exceptional points contain no blanks.) We are now ready to prove that for sufficiently large $k \in \mathbb{N}, X=\widetilde{X}_{k}$ satisfies the claims of Theorem 4.2 .

Proof. (10) In $X_{k}$, any level- $n$ square has shape $\left[\overrightarrow{\mathbb{1}},\left(2^{n+1}+2^{n-1}-2\right) \overrightarrow{\mathbb{1}}\right]$ and contains $4^{n-1}$ blank tiles, so the density of blank $(\square)$ tiles in any $x_{\omega} \in X_{k}\left(\omega \in \mathcal{T}^{\mathbb{N}}\right)$ is

$$
f_{\square}:=\lim _{n \rightarrow \infty} \frac{4^{n-1}}{\left(2^{n+1}+2^{n-1}-2\right)^{2}}=\frac{1}{25} .
$$

Therefore, since any blank tile may have $k$ labels, it is not hard to check that $h\left(X_{k}\right)=\lim _{n \rightarrow \infty} \frac{\log \left|\mathcal{L}_{\left[\overrightarrow{1},\left(2^{n+1}+2^{n-1}-2\right) \overrightarrow{1}\right]}\left(X_{k}\right)\right|}{\left(2^{n+1}+2^{n-1}-2\right)^{2}} \geq \frac{\log k}{25}$ for any positive integer $k \in \mathbb{N}$. Since $X_{k} \subsetneq \widetilde{X}_{k}$, certainly $h\left(\widetilde{X}_{k}\right) \geq h\left(X_{k}\right) \geq \frac{\log k}{25}$. So we may set $X=\widetilde{X}_{k}$ with $k$ chosen large enough that $h(X)>M$.

(21) (4) These claims may be proved just as they were proved in the case $d \geq 3$. (Recall that for any $x \in X$ and $n \in \mathbb{N}$, there will exist $l \in \mathbb{N}$ and some rectangular 
block $B:=[\vec{v}, \vec{w}]$ such that $[\vec{v}+l \overrightarrow{\mathbb{1}}, \vec{w}-l \overrightarrow{\mathbb{1}}]$ contains $[-n \overrightarrow{\mathbb{1}}, n \overrightarrow{\mathbb{1}}]$ and for all $\vec{\imath}$ in the thick border $[\vec{v}, \vec{w}] \backslash[\vec{v}+l \overrightarrow{\mathbb{1}}, \vec{w}-l \overrightarrow{\mathbb{1}}]$, the symbol $x_{\vec{\imath}}$ is an arrow.)

(6) It is shown in Theorem 6.23 of [12] that $\widetilde{X}_{1}$ is topologically mixing, and the proof extends trivially to any $\widetilde{X}_{k}$.

To prove $X$ has top.c.p.e., assume that $\phi: X \rightarrow Y$ is a sliding block code from $X$ onto $Y$, and denote by $2 m+1\left(m \in \mathbb{N}_{0}\right)$ the size of its coding window. If $Y$ is non-trivial, then there exist two patterns $P, P^{\prime} \in \mathcal{L}_{[-m \overrightarrow{\mathbb{1}}, m \overrightarrow{\mathbb{1}}]}(X)$ so that $\phi(P) \neq \phi\left(P^{\prime}\right)$. By definition of transitivity, any transitive point of $X$ contains both $P$ and $P^{\prime}$ as subpatterns, and by the description of the transitive points in Lemma 6.16 of [12, for some $n \in \mathbb{N}$ there exists a level- $n$ rectangle $Q_{n}$ whose dimensions are $a_{n} \times b_{n}\left(a_{n}, b_{n} \in \mathbb{N}\right)$ and which contains both $P$ and $P^{\prime}$ as subpatterns. Say that $\left.Q_{n}\right|_{\vec{\imath}+[-m \overrightarrow{\mathbb{1}}, m \overrightarrow{\mathbb{1}}]}=P$ and $\left.Q_{n}\right|_{\vec{\jmath}+[-m \overrightarrow{\mathbb{1}}, m \overrightarrow{\mathbb{1}}]}=P^{\prime}$ for some $\vec{\imath} \neq \vec{\jmath} \in \mathbb{Z}^{2}$.

We claim that for $N \in \mathbb{N}$ large enough there exist two level- $N$ rectangles $Q_{N}, Q_{N}^{\prime}$ both having a border colored $S E$, both of dimensions $a_{N} \times b_{N}\left(a_{N}, b_{N} \in \mathbb{N}\right)$ and such that $\left.Q_{N}\right|_{B}=P$ and $\left.Q_{N}^{\prime}\right|_{B}=P^{\prime}$ for some fixed $B \subseteq\left[\overrightarrow{\mathbb{1}},\left(a_{N}, b_{N}\right)\right] \subsetneq \mathbb{Z}^{2}$ a translated copy of $[-m \overrightarrow{\mathbb{1}}, m \overrightarrow{\mathbb{1}}]$. To prove this it suffices to show that we can decrease the horizontal $\left|\vec{\jmath}_{1}-\vec{\imath}_{1}\right|$ and vertical $\left|\vec{\jmath}_{2}-\vec{\imath}_{2}\right|$ distance between occurrences of $P$ and $P^{\prime}$ in $Q_{n}$ by increasing the level of the rectangle.

Without loss of generality, assume that $\vec{\jmath}_{1}>\vec{\imath}_{1}$ and that the border of $Q_{n}$ is colored $S E$. It is shown in 12 that for any $a, b \in I_{n}:=\left[2^{n+1}+2^{n-1}-2,2^{n+1}+\right.$ $\left.2^{n}-2\right] \cap \mathbb{Z}$, there exists a level- $n$ rectangle with dimensions $a \times b$. We now construct two level- $(n+1)$ rectangles $Q_{n+1}$ and $Q_{n+1}^{\prime}$. $Q_{n+1}$ has a border colored $N W$, and the four level- $n$ rectangles in it have the following properties: the northwest rectangle has dimensions $a \times a$ for some allowed integers $a, a+1 \in I_{n}$, the southeast rectangle is $Q_{n}$, and the northeast and southwest rectangles are any with the correct dimensions, i.e. the northeast is $a_{n} \times a$ and the southwest is $a \times b_{n} . Q_{n+1}^{\prime}$ is described in exactly the same way as $Q_{n+1}$, except that the northwest subrectangle of $Q_{n+1}^{\prime}$ has dimensions $a+1 \times a$. The dimensions of $Q_{n+1}$ are then $a+a_{n}+2 \times a+b_{n}+2$, and the dimensions of $Q_{n+1}^{\prime}$ are $a+a_{n}+3 \times a+b_{n}+2$. We finally create two level$(n+2)$ rectangles $Q_{n+2}$ and $Q_{n+2}^{\prime}$ in a similar fashion. $Q_{n+2}$ has a border colored $S E$, and the four level- $(n+1)$ rectangles in it have the following properties: the northwest rectangle is $Q_{n+1}$, the southeast rectangle has dimensions $b+1 \times b+1$ for some allowed integers $b, b+1 \in I_{n+1}$, and the northeast and southwest rectangles are any with the correct dimensions. $Q_{n+2}^{\prime}$ is described in exactly the same way as $Q_{n+2}$, except that the southeast subrectangle of $Q_{n+2}^{\prime}$ has dimensions $b \times b+1$. Then, $Q_{n+2}$ and $Q_{n+2}^{\prime}$ both have dimensions $a_{n}+a+b+5 \times b_{n}+a+b+5$. Also, it is not hard to see that there exists $B^{\prime} \subseteq\left[\overrightarrow{\mathbb{1}},\left(a_{n}+a+b+5, b_{n}+a+b+5\right)\right] \subsetneq \mathbb{Z}^{2}$ a translated copy of $[-m \overrightarrow{\mathbb{1}}, m \overrightarrow{\mathbb{1}}]$ so that $\left.Q_{n+2}\right|_{\vec{\imath}+B^{\prime}}=P$ and $\left.Q_{n+2}^{\prime}\right|_{\vec{\jmath}+B^{\prime}-\vec{e}_{1}}=P^{\prime}$, so the horizontal distance between subpatterns $P$ and $P^{\prime}$ has decreased by one. By repeating this procedure, we will eventually arrive at a pair $Q_{n^{\prime}}, Q_{n^{\prime}}^{\prime}$ of level- $n^{\prime}$ rectangles $\left(n^{\prime}>n\right)$, both with dimensions $a_{n^{\prime}} \times b_{n^{\prime}}\left(a_{n^{\prime}}, b_{n^{\prime}} \in \mathbb{N}\right)$ such that $P$ appears in $Q_{n^{\prime}}$ at the same $\vec{e}_{1}$-coordinate as $P^{\prime}$ in $Q_{n^{\prime}}^{\prime}$. Similarily, we may decrease the (vertical) $\vec{e}_{2}$-coordinate, changing the role of the integers $a$ and $a+1$, resp. $b$ and $b+1$. After finitely many steps we arrive at level- $N$ rectangles $Q_{N}, Q_{N}^{\prime}$ as claimed.

Now for any $N^{\prime}>N$, it is possible to construct a level- $N^{\prime}$ rectangle such that all $4^{N^{\prime}-N}$ of its level- $N$ subrectangles have dimensions $a_{N} \times b_{N}$. The dimensions of 
this level- $N^{\prime}$ rectangle are then $2^{N^{\prime}-N} a_{N}+2^{N^{\prime}-N+1}-2 \times 2^{N^{\prime}-N} b_{N}+2^{N^{\prime}-N+1}-2$. Obviously each of the $4^{N^{\prime}-N-1}$ level- $N$ subrectangles having a border colored $S E$ can be independently filled either by $Q_{N}$ or by $Q_{N}^{\prime}$. As $\left.Q_{N}\right|_{B}=P$ and $\left.Q_{N}^{\prime}\right|_{B}=P^{\prime}$ we know that $\phi\left(Q_{N}\right) \neq \phi\left(Q_{N}^{\prime}\right)$, and hence all of the $2^{4^{N^{\prime}-N-1}}$ level- $N^{\prime}$ rectangles createable in this manner have distinct $\phi$-images. This means that the number of distinct $\left(2^{N^{\prime}-N} a_{N}+2^{N^{\prime}-N+1}-2-2 m\right) \times\left(2^{N^{\prime}-N} b_{N}+2^{N^{\prime}-N+1}-2-2 m\right)$ patterns in the language of $Y$ is at least $2^{4^{N^{\prime}-N-1}}$, and so the entropy of $Y$ will be

$$
\begin{gathered}
h(Y) \geq \lim _{N^{\prime} \rightarrow \infty} \frac{\log \left(2^{4^{N^{\prime}-N-1}}\right)}{\left(2^{N^{\prime}-N} a_{N}+2^{N^{\prime}-N+1}-2-2 m\right)\left(2^{N^{\prime}-N} b_{N}+2^{N^{\prime}-N+1}-2-2 m\right)} \\
=\frac{\log 2}{4 a_{N} b_{N}+8\left(a_{N}+b_{N}\right)+16}>0 .
\end{gathered}
$$

It follows that every non-trivial subshift factor of $X$ has positive entropy, and therefore no non-trivial topological factor of $X$ has zero entropy.

Remark 9.1. Our techniques allow some give and take in the properties enjoyed by an example. Above, if we set $X$ equal to $X_{k}$ rather than $\widetilde{X}_{k}$, then $X$ would not be mixing and $X$ would not be top.c.p.e. However, $X=X_{k}$ would still satisfy conditions (11)-(4) of Theorem 4.2. Also (in contrast to $\widetilde{X}_{k}$ ), $X_{k}$ would satisfy (5) (i.e. $X_{k}$ has no topological factor which admits a c.p.e. measure); this follows from Proposition 6.1 because $X_{k}$ has the zero entropy factor $X_{1}$ and arbitrarily large blocks from the zero entropy exceptional shift $E$ occur in every point of $X_{k} \cdot X_{k}$ would also have a top.c.p.e. sofic factor (constructed from $X_{k}$ by sending all nonblank symbols and one of the blank symbols to 0 , and leaving the other $k-1$ blank symbols unchanged).

Alternately, using the technique which built the gently ascending hyperplanes in Section 7 . we could build an SFT $\widetilde{X}_{k}^{*}$ by superimposing on $\widetilde{X}_{k}$ an SFT which allows the blocks of blanks with sizes $2 \times 2,1 \times 2,2 \times 1$ to occur only very rarely, so that $h\left(\widetilde{X}_{1}^{*}\right)=0$ even though the blank symbol will occur with positive frequency in some points. Now $\widetilde{X}_{k}^{*}$ is mixing and has no topological factor admitting a c.p.e. invariant measure, and it satisfies the other statements of Theorem 4.2, with the following exception: $\widetilde{X}_{k}^{*}$ has no factor which is top.c.p.e.

\section{A TOpologically MiXing, TOP.C.P.E. $\mathbb{Z}^{t}$ SOFiC EXAMPLE}

In this section we complete the proof of Theorem 4.1 by establishing the case $d=2$.

Proof. Let $X=\widetilde{X}_{k}$ be the $\mathbb{Z}^{2}$ SFT constructed in the previous section, satisfying the statement of Theorem 4.2 in the case $d=2$. We define $S$ as the image of $X$ under the one-block factor map $\pi$ which sends all non-blank symbols in the alphabet of $\widetilde{X}_{k}$ to 0 and leaves each of the $k$ blank symbols unchanged. Hence $S$ is a subshift with an alphabet $\widetilde{\mathcal{A}}_{k}:=\{0\} \cup\left\{\square_{j} \mid 1 \leq j \leq k\right\}$ and $\pi$ collapses the subsystem of exceptional points $E \subsetneq \widetilde{X}_{k}$ to a single (fixed) point. We now go on to verify the claims of Theorem 4.1

(11), (7) We claim that $h(X)=h(S)$. As $S$ is a factor of $X$ we only need to show $h(X) \leq h(S)$. For this we estimate the number of patterns in $\mathcal{L}_{B_{n}}(X)$ in terms of image patterns in $\mathcal{L}_{B_{n}}(S)$ for $B_{n}:=[\overrightarrow{\mathbb{1}}, n \overrightarrow{\mathbb{1}}](n \in \mathbb{N})$. Preimages of the 
pattern $0^{B_{n}} \in \mathcal{L}_{B_{n}}(S)$ are subpatterns of exceptional points, and their number is thus bounded by $\left|\mathcal{A}_{k}\right|^{4 n-4}$ as before (the symbols along the boundary determine the interior). Every other pattern $P \in \mathcal{L}_{B_{n}}(S)$ contains at least one blank, and every preimage can be recreated from an $n$-tuple of colors $t \in \mathcal{T}^{n}$ as follows: let $s \in S$ such that $\left.s\right|_{B_{n}}=P$. Starting at the lexicographically smallest coordinate $\vec{\imath} \in B_{n}$ containing a blank, surround it (and the adjacent blanks if any) by a level-1 border of color $t_{1}$. Knowing $t_{1}$, we can also put down the three neighboring level-1 rectangles. In doing so, we completed the interior of the level-2 rectangle containing the blank at $\vec{\imath}$; now surround it by a border of arrows of color $t_{2}$. Continue in this way: for any $i>2$, take the next color $t_{i}(1<i \leq n)$ from the list and surround the already placed level- $(i-1)$ rectangles with a level- $i$ border of color $t_{i}$ (we can easily determine its size from the knowledge of the positions of the blanks) and fill in all remaining symbols in its interior (all 4 subrectangles have to be level- $(i-1))$. In every step the region already colored extends at least 1 coordinate further into each direction than in the step before, so after at most $n$ steps we have completely filled $B_{n}$. As every preimage of $P$ can be obtained by this procedure, $\left|\pi^{-1}(P)\right| \leq 4^{n} \leq\left|\mathcal{A}_{k}\right|^{4 n}$ and we have $\left|\mathcal{L}_{B_{n}}(X)\right| \leq\left|\mathcal{A}_{k}\right|^{4 n} \cdot\left|\mathcal{L}_{B_{n}}(S)\right|$. Hence $h(X) \leq h(S)$, proving our claim and thus (7). (1) follows because $h(X)>M$.

(2) Because $E$ contains every minimal subsystem of $X$, the image of $E$ (which is the fixed point) contains every minimal subsystem of $S$.

(3), (44) These items are proved as in the case $d \geq 3$. (Note that finite configurations must occur inside blocks with arbitrarily large neighborhoods of the boundary colored 0.)

(66), (8) $S$ is mixing and top.c.p.e. because it is a non-trivial factor of $X$ which has those properties. Trivially (6) also follows.

Remark 10.1. Let $\widetilde{S}_{k}^{*}$ be the sofic shift which is the image under the map $\pi$ above of the SFT $\widetilde{X}_{k}^{*}$ described in Remark 9.1. This shift is mixing and satisfies the conditions satisfied by $S$ above, except that it has no top.c.p.e. factor. However, no topological factor of $\widetilde{S}_{k}^{*}$ admits a c.p.e. invariant measure.

\section{Appendix A. A hierarchy of mixing notions for $\mathbb{Z}^{d}$ Shifts}

In this appendix, we collect and put into a more general framework some mixing properties for $\mathbb{Z}^{d}$ shifts dispersed throughout the literature [9, 14, 16, 17, 24, 25, 27, as well as the block gluing notion (3.1) of the current paper. We record some known and some new facts about these mixing properties. We show that all of them can be put into a linear chain, in which - with one possible exception - none of the logical implications can be reversed (A.6).

Recall that $\delta_{\infty}$ represents the maximum-metric on $\mathbb{Z}^{d}$, i.e. $\delta_{\infty}(\vec{v}, \vec{w})=\|\vec{v}-\vec{w}\|_{\infty}$ $:=\max _{1 \leq k \leq d}\left|v_{k}-w_{k}\right|$, and also its natural extension to a separation function on non-empty subsets $V, W \subseteq \mathbb{Z}^{d}: \delta_{\infty}(V, W):=\min _{\vec{v} \in V, \vec{w} \in W} \delta_{\infty}(\vec{v}, \vec{w})$.

We start with two common definitions from the $\mathbb{Z}^{d}$ shift literature.

Definition A.1. A $\mathbb{Z}^{d}$ shift $X$ is topologically mixing if for any two non-empty finite subsets $V, W \subsetneq \mathbb{Z}^{d}$ there exists a constant $D_{V, W} \in \mathbb{N}_{0}$ so that for any $\vec{\imath} \in \mathbb{Z}^{d}$ such that $\delta_{\infty}(V, W+\vec{\imath})>D_{V, W}$, any pair of patterns on $V$ and $W+\vec{\imath}$ occurring in $X$ can be put together to form a valid point of $X$, i.e. $\forall x, y \in X, \exists z \in X$ such that $\left.z\right|_{V}=\left.x\right|_{V}$ and $\left.z\right|_{W+\vec{\imath}}=\left.y\right|_{W+\vec{\imath}}$. 
Furthermore, remember that $\vec{v} \preceq \vec{w}$ is shorthand for the fact that the components of $\vec{v}, \vec{w} \in \mathbb{Z}^{d}$ satisfy the inequality $\vec{v}_{k} \leq \vec{w}_{k}$ for all $1 \leq k \leq d$. As in Section 3. the notation $B=[\vec{v}, \vec{w}]:=\left\{\vec{\imath} \in \mathbb{Z}^{d} \mid \vec{v} \preceq \vec{\imath} \preceq \vec{w}\right\}$ will be used for solid blocks in $\mathbb{Z}^{d}$ and $\overrightarrow{\mathbb{1}}$ refers to the element in $\mathbb{Z}^{d}$ in which all components equal 1.

Definition A.2 ([14]). A $\mathbb{Z}^{d}$ shift $X$ is corner gluing at gap $g \in \mathbb{N}_{0}$ if for any $\vec{u}, \vec{v}, \vec{w} \in \mathbb{Z}^{d}$ with $\vec{u}+\overrightarrow{\mathbb{1}} \preceq \vec{v} \preceq \vec{w}-g \overrightarrow{\mathbb{1}}$, any patterns on $L:=[\vec{u}, \vec{w}] \backslash[\vec{v}, \vec{w}]-$ which we will call the corner-shape - and a solid block $R:=[\vec{v}+g \overrightarrow{\mathbb{1}}, \vec{w}]$ which are legal in $X$ can be put together in the same point of $X$, i.e. $\forall x, y \in X, \exists z \in X$ such that $\left.z\right|_{L}=\left.x\right|_{L}$ and $\left.z\right|_{R}=\left.y\right|_{R}$.

Next we introduce the general concepts of $\mathcal{F}$-gluing and $\mathcal{F}$-filling and exhibit some special cases of these new notions which are already present in the literature.

Definition A.3. Let $\mathcal{F} \subseteq\left\{F \subsetneq \mathbb{Z}^{d} \mid F \neq \emptyset \wedge F\right.$ finite $\}$ be a family of non-empty finite subsets in $\mathbb{Z}^{d}$.

A $\mathbb{Z}^{d}$ shift $X$ is called $\mathcal{F}$-gluing at gap $g \in \mathbb{N}_{0}$ if, for any two elements $V, W \in \mathcal{F}$ with separation $\delta_{\infty}(V, W)>g$ and any two patterns on $V$ and $W$ which occur in $X$, there is a valid point in $X$ realizing these two patterns, i.e. $\forall x, y \in X, \exists z \in X$ such that $\left.z\right|_{V}=\left.x\right|_{V}$ and $\left.z\right|_{W}=\left.y\right|_{W}$.

Similarily a $\mathbb{Z}^{d}$ shift $X$ is called $\mathcal{F}$-filling at gap $g \in \mathbb{N}_{0}$ if for any element $V \in \mathcal{F}$ and any (possibly infinite) subset $\emptyset \neq W \subseteq \mathbb{Z}^{d}$ with separation $\delta_{\infty}(V, W)>g$ every pair of patterns on $V$ and $W$ which occur in $X$ can be put together to form a valid point in $X$, i.e. $\forall x, y \in X, \exists z \in X$ such that $\left.z\right|_{V}=\left.x\right|_{V}$ and $\left.z\right|_{W}=\left.y\right|_{W}$.

A $\mathbb{Z}^{d}$ shift $X$ is called $\mathcal{F}$-gluing, resp. $\mathcal{F}$-filling, if it is $\mathcal{F}$-gluing at gap $g$, resp. $\mathcal{F}$-filling at gap $g$, for some $g \in \mathbb{N}_{0}$.

Observation A.4. Note that by compactness the notions of $\mathcal{F}$-gluing at gap $g$ and $\mathcal{F}$-filling at gap $g$ coincide for $\mathcal{F}=\left\{F \subsetneq \mathbb{Z}^{d} \mid F \neq \emptyset \wedge F\right.$ finite $\}$ the collection of all finite subsets. $\mathbb{Z}^{d}$ shifts satisfying this complete gluing/filling condition are usually (following [6]) called strongly irreducible (SI), and we will use this terminology for the two equivalent properties.

However, for general sets $\mathcal{F}$ the notions of $\mathcal{F}$-gluing and $\mathcal{F}$-filling need not coincide, as we can check with the following:

Observation A.5. For $\mathcal{B}:=\left\{B=[\vec{v}, \vec{w}] \mid \vec{v} \preceq \vec{w} \in \mathbb{Z}^{d}\right\}$ the collection of all solid blocks, the notion $\mathcal{B}$-gluing at gap $g$ becomes block gluing at gap $g$ as defined in Section 3 .

The property of being $\mathcal{B}$-filling already appears in the literature as the uniform filling property (UFP). For simplicity we will also use this common expression. As we will see in Theorem A.6, block gluing and having the UFP (i.e. being block filling) are not equivalent for $\mathbb{Z}^{d}$ shifts.

Theorem A.6. For $g \in \mathbb{N}_{0}$ and $a \mathbb{Z}^{d}$ shift $X$ we have the following implications. $X$ is SI at gap $g \stackrel{(1)}{\Longrightarrow} X$ has the UFP at gap $g \stackrel{(2)}{\Longrightarrow} X$ is corner gluing at gap $g \stackrel{(3)}{\Longrightarrow}$ $X$ is block gluing at gap $g \stackrel{(4)}{\Longrightarrow} X$ is topologically mixing. Implications (2), (3), and (4) cannot be reversed even if we allow an increase in $g$.

Proof. Implications (1), (2) and (4) are fairly clear from the definitions.

We prove implication (3). Let the $\mathbb{Z}^{d}$ shift $X$ be corner gluing at gap $g \in \mathbb{N}_{0}$ and let $B_{1}=\left[\vec{v}^{(1)}, \vec{w}^{(1)}\right], B_{2}=\left[\vec{v}^{(2)}, \vec{w}^{(2)}\right] \subsetneq \mathbb{Z}^{d}$ be two disjoint solid blocks with 
separation $\delta_{\infty}\left(B_{1}, B_{2}\right)>g$. There must be some $1 \leq j \leq d$ such that the intervals $\left[\left(\vec{v}^{(1)}\right)_{j},\left(\vec{w}^{(1)}\right)_{j}\right],\left[\left(\vec{v}^{(2)}\right)_{j},\left(\vec{w}^{(2)}\right)_{j}\right] \subsetneq \mathbb{Z}$ have separation larger than $g$; otherwise, since each $B_{i}(i=1,2)$ is a product $\prod_{1 \leq k \leq d}\left[\left(\vec{v}^{(i)}\right)_{k},\left(\vec{w}^{(i)}\right)_{k}\right]$, we could find points in $B_{1}$ and $B_{2}$ at a distance at most $g$. Without loss of generality, suppose $\left(\vec{w}^{(1)}\right)_{j}+$ $g<\left(\vec{v}^{(2)}\right)_{j}$. Define $L:=\mathbb{Z}^{d} \cap\left(\prod_{k=1}^{d}\left[\min \left\{\vec{v}_{k}^{(1)}, \vec{v}_{k}^{(2)}\right\}-g-1, \max \left\{\vec{w}_{k}^{(1)}, \vec{w}_{k}^{(2)}\right\}\right]\right) \backslash$ $\left(\prod_{k=1}^{d}\left[\vec{v}_{k}^{(2)}-g, \max \left\{\vec{w}_{k}^{(1)}, \vec{w}_{k}^{(2)}\right\}\right]\right)$ and $R:=\mathbb{Z}^{d} \cap \prod_{k=1}^{d}\left[\vec{v}_{k}^{(2)}, \max \left\{\vec{w}_{k}^{(1)}, \vec{w}_{k}^{(2)}\right\}\right]$. By corner gluing, any patterns on $L$ and $R$ which are legal in $X$ can occur in the same point. Because $B_{1} \subseteq L$ and $B_{2} \subseteq R$, the same holds for $B_{1}$ and $B_{2}$.

It will be shown in Example C.1 that corner gluing (at gap 2) does not imply the UFP, and so implication (2) cannot be reversed.

Block gluing at gap 1 does not imply corner gluing, as will be shown in Example A.7 below. Therefore, implication (3) cannot be reversed.

Finally, we will see in Appendix B that non-trivial block gluing $\mathbb{Z}^{d}$ shifts have positive entropy (B.2), and so since there exist topologically mixing shifts with zero entropy (for instance, those in 24), topological mixing does not imply block gluing at any gap either. Thus implication (4) cannot be reversed.

We point out that whether or not SI and UFP define the same notion still seems to be an open question.

The following example shows that the condition of being block gluing is in fact strictly weaker than corner gluing.

Example A.7. Consider the SFT $X \subsetneq\{0,1\}^{\mathbb{Z}^{2}}$ with forbidden pattern $Q=\begin{array}{ll}0 & 1 \\ 0 & 0\end{array}$. In other words, $x \in\{0,1\}^{\mathbb{Z}^{2}}$ is in $X$ if and only if $x$ does not contain $Q$ as a subword. We claim that $X$ is block gluing at gap 1 , but not corner gluing at any gap.

Take any pair of solid blocks $B_{1}, B_{2} \subsetneq \mathbb{Z}^{2}$ with $\delta_{\infty}\left(B_{1}, B_{2}\right)>1$, and any pair of patterns $P_{1} \in \mathcal{L}_{B_{1}}(X)$ on $B_{1}$ and $P_{2} \in \mathcal{L}_{B_{2}}(X)$ on $B_{2}$. We claim that the point $x \in\{0,1\}^{\mathbb{Z}^{2}}$ defined by $\left.x\right|_{B_{1}}:=P_{1},\left.x\right|_{B_{2}}:=P_{2}$, and $x_{\vec{\imath}}:=1$ for all $\vec{\imath} \notin B_{1} \cup B_{2}$ is in $X$. All that must be checked is that $x$ does not contain $Q$ as a subword. Consider any $2 \times 2$ solid block $B \subsetneq \mathbb{Z}^{2}$. There are two cases. If $B \subseteq B_{1}$ or $B \subseteq B_{2}$, then $\left.x\right|_{B}$ is a subword of $P_{1}$ or $P_{2}$ and therefore does not equal $Q$. If $B \nsubseteq B_{1}$ and $B \nsubseteq B_{2}$, then $\left|B \backslash\left(B_{1} \cup B_{2}\right)\right| \geq 2$. Therefore, $\left.x\right|_{B}$ contains at least two 1s and is again not equal to $Q$. So, $x$ does not contain $Q$ as a subword, and $x \in X$. This means that $X$ is block gluing at gap 1 .

Now consider, for any $g \in \mathbb{N}_{0}$, the solid blocks $B_{1}=[\overrightarrow{0},(g+1) \overrightarrow{\mathbb{1}}], B_{2}=[\overrightarrow{\mathbb{1}},(g+$ 1) $\overrightarrow{\mathbb{1}}]$, and define the set $L:=B_{1} \backslash B_{2} \subsetneq \mathbb{Z}^{2}$. The pattern $P:=0^{L}$ on $L$ consisting of all $0 \mathrm{~s}$ is certainly valid for $X$; for instance, it is a subword of the point $0^{\mathbb{Z}^{2}}$, which is in $X$. However, we claim that if $P$ appears in some point $x \in X$, i.e. there exists $\vec{\imath} \in \mathbb{Z}^{2}$ so that $\left.x\right|_{\vec{\imath}+L}=P$, this actually forces the entire solid block on whose lower-left boundary the pattern $P$ occurs to be filled with 0 s, i.e. $\left.x\right|_{\vec{\imath}+B_{1}}=0^{B_{1}}$. Suppose this is not the case. Then there would exist $x \in X$ and $\vec{\imath} \in \mathbb{Z}^{2}$ so that $\left.x\right|_{\vec{\imath}+L}=P$ and $\left.x\right|_{\vec{\imath}+B_{1}}$ contains a 1 . Consider the lowest row of $\left.x\right|_{\vec{\imath}+B_{1}}$ containing a 1 and then take the leftmost 1 in this row. The $2 \times 2$ solid block with this 1 in its upper-right corner will have $0 \mathrm{~s}$ in its other locations, and so equals $Q$. This cannot happen since $x \in X$, so we have a contradiction and in fact $\left.x\right|_{\vec{\imath}+B_{1}}$ has all 0 s. Now define the singleton set $R:=\{(g+1) \overrightarrow{\mathbb{1}}\} \subsetneq \mathbb{Z}^{2}$, and let the pattern on $R$ be just a single 1 . Certainly the pattern 1 appears in $X$, e.g. in the point $1^{\mathbb{Z}^{2}}$. However, there does not exist $x \in X$ so that $\left.x\right|_{L}=P$ and $\left.x\right|_{R}=1$. As $\delta_{\infty}(L, R)>g$ this 
means that $X$ is not corner gluing at gap $g$, and since $g$ was arbitrary, $X$ is not corner gluing (at any gap size).

\section{Appendix B. Block gluing $\mathbb{Z}^{d}$ Shifts}

In this section we consider some consequences of the very natural block gluing condition, some of which are used in the proof of Theorems 3.2. 4.1 and 4.2.

Lemma B.1. Let $d \geq 1, g \in \mathbb{N}_{0}$ and let $X$ be a $\mathbb{Z}^{d}$ shift. Then the following statements are equivalent.

(1) $X$ is block gluing at gap $g$.

(2) For $n \in \mathbb{N}_{0}$ and any countable family $\left(P_{\vec{\imath}} \in \mathcal{L}_{[\overrightarrow{\mathbb{1}}, n \overrightarrow{\mathbb{1}}]}(X)\right)_{\vec{\imath} \in(g+n) \mathbb{Z}^{d}}$ of individually valid patterns there exists a point $x \in X$ such that $\left.x\right|_{\vec{\imath}+[\overrightarrow{\mathbb{1}}, n \overrightarrow{\mathbb{1}}]}=P_{\vec{\imath}}$ for all $\vec{\imath} \in(g+n) \mathbb{Z}^{d}$.

Proof. (2) $\Longrightarrow(1)$ is trivial, and the converse is a straightforward compactness argument.

Theorem B.2. Suppose $d \geq 1$ and let $X$ be a non-trivial block gluing $\mathbb{Z}^{d}$ shift. Then the following hold.

(1) $h(X)>0$.

(2) Every subshift factor of $X$ is again block gluing.

(3) $X$ has topologically completely positive entropy.

(4) For every $\varepsilon>0, X$ contains a proper subshift $W \subsetneq X$ such that $h(W)>$ $h(X)-\varepsilon$.

(5) For every non-empty proper subsystem $V \subsetneq X$ and every $\varepsilon>0$ there is a non-empty subshift $W \subsetneq X$ disjoint from $V$ with $h(W)>h(X)-\varepsilon$.

Proof. Assume that $X$ is block gluing at gap $g \in \mathbb{N}_{0}$.

(11) This follows easily from condition (2) of Lemma B.1

(21), (3) Suppose $\phi: X \rightarrow Y$ is a factor map between subshifts, such that $\left.x\right|_{[-n \overrightarrow{\mathbb{1}}, n \overrightarrow{\mathbb{1}}]}\left(n \in \mathbb{N}_{0}\right)$ determines $(\phi(x))_{0}$. Patterns on $Y$-blocks with separation $g+2 n$ are the images of patterns on $X$-blocks with separation $g$, and therefore $Y$ is block gluing at gap $g+2 n$. (3) now follows from (11) and (2).

(5) Let $P \in \mathcal{L}(X) \backslash \mathcal{L}(V)$ be a pattern appearing in some point in $X$ but not in any point of the proper subsystem $V$; w.l.o.g. we assume $P \in \mathcal{L}_{[\overrightarrow{\mathbb{1}}, n \overrightarrow{\mathbb{1}}]}(X)$ for some fixed $n \in \mathbb{N}$. Let $\mathcal{P}_{N}:=\left\{Q \in \mathcal{L}_{[\overrightarrow{\mathbb{1}}, N \overrightarrow{\mathbb{1}}]}(X)|Q|_{[\overrightarrow{\mathbb{1}}, n \overrightarrow{\mathbb{1}}]}=P\right\}$ with $N \geq n$. By block gluing, every element of $\mathcal{L}_{[\overrightarrow{\mathbb{1}},(N-n-g) \overrightarrow{\mathbb{1}}]}(X)$ occurs as the upper corner subblock of some element of $\mathcal{P}_{N}$, so $\left|\mathcal{P}_{N}\right| \geq\left|\mathcal{L}_{[\overrightarrow{\mathbb{1}},(N-n-g) \overrightarrow{\mathbb{1}}]}(X)\right|$. Given $N>g$, let $B:=[\overrightarrow{\mathbb{1}},(2 N+g) \overrightarrow{\mathbb{1}}]$ and define a subshift

$W_{N}:=\left\{x \in X \mid \forall \vec{\imath} \in \mathbb{Z}^{d}\right.$ : the block $\left.x\right|_{\vec{\imath}+B}$ contains the subpattern $\left.P\right\}$.

Clearly $W_{N}$ is disjoint from $V$. By condition (2) of Lemma B.1, patterns from $\mathcal{P}_{N}$ may appear independently on the lattice $(g+N) \mathbb{Z}^{d}$ in points of $X$. Such points are in $W_{N}$, and it follows that $\lim _{N \rightarrow \infty} h\left(W_{N}\right)=h(X)$.

(4) We will show that $X$ contains a proper subshift, and then (5) will imply (4). Given $N \in \mathbb{N}$, pick a pattern $P \in \mathcal{L}_{[\overrightarrow{\mathbb{1}}, N \overrightarrow{\mathbb{1}}]}(X)$, and let $U_{N}$ be the set of points $x$ in $X$ such that $\left.x\right|_{\vec{\imath}+[\overrightarrow{\mathbb{1}}, N \overrightarrow{\mathbb{1}}]}=P$ for all $\vec{\imath} \in(g+N) \mathbb{Z}^{d}$. By condition (2) of Lemma B.1. $U_{N}$ is non-empty. Also $U_{N}$ is closed, and invariant under shifts from $N \mathbb{Z}^{d}$. Thus the $\mathbb{Z}^{d}$ shift orbit closure $V_{N}$ of $U_{N}$ is the union of images of $U_{N}$ under finitely 
many (i.e. $N^{d}$ ) shifts. Clearly $\lim _{N \rightarrow \infty} h\left(V_{N}\right)=0$, and for large $N$ it follows that $V_{N}$ is a proper subshift of $X$.

We are interested in the implications of block gluing (or other mixing conditions) on richness and separation properties of subsystems. Within a mixing $\mathbb{Z} \mathrm{SFT} X$ of positive entropy, any two disjoint subsystems are contained in disjoint mixing subSFTs of $X$, with entropy arbitrarily close to that of $X$. This technical fact is tremendously useful for constructions with $\mathbb{Z}^{d}$ SFTs when $d=1$ (e.g. see 7]), but it fails for $d>1$ [24]. As part of the effort to understand the boundaries of how properties do and do not extend to higher-dimensional SFTs, we ask the following.

Question B.3. Given $\varepsilon>0$ and $d \geq 2$, is any pair of disjoint subsystems $V_{1}, V_{2}$ inside a block gluing $\mathbb{Z}^{d}$ SFT $X$ contained in a pair $W_{1} \supseteq V_{1}, W_{2} \supseteq V_{2}$ of disjoint block gluing subSFTs of entropy at least $h(X)-\varepsilon$ ?

For $d=1$, the block gluing SFTs are the mixing SFTs, and the answer to Question B.3 is well known to be yes 7, Section 26].

For $d=1,2$ (but not for $d \geq 3$ ), it is known that finite orbits of a $\mathbb{Z}^{d}$ strongly irreducible SFT are dense [30. The same argument works for block gluing SFTs, and we have the same question.

Question B.4. Are there block gluing $\mathbb{Z}^{d}$ SFTs (for $d \geq 3$ ) that do not have dense periodic points? Are there examples with no periodic points?

From 24] we know that every $\mathbb{Z}^{d}$ SFT having the UFP is entropy minimal (i.e., every proper subsystem has strictly smaller entropy). We note that Example C.1 provides the existence of non-trivial block gluing $\mathbb{Z}^{d}$ SFTs $(d \geq 2)$ which are not entropy minimal.

\section{Appendix C. A non-entropy-minimal 4-Corner-Gluing $\mathbb{Z}^{2}$ SFT}

This appendix contains a $\mathbb{Z}^{2}$ SFT which is corner gluing in all 4 corners, i.e. in the NW-, NE-, SW- and SE-corners, but which still does not have the UFP. With a small adjustment we can even violate entropy minimality. In particular this example proves that implication (2) in Theorem A.6 cannot be reversed.

The $\mathbb{Z}^{2}$ SFT we construct models a system of meandering streams running in a $\mathbb{Z}^{2}$ plane that has a large slope in the north-south direction, but no slope in the east-west direction. Therefore a stream starting somewhere north has to constantly run south (it will never run uphill), with some possible meandering in the east-west direction. It can split into different "branches", and those branches can unify again. However there are neither sources nor sinks, thus a stream has to run forever without starting or ending at a certain coordinate in $\mathbb{Z}^{2}$.

Example C.1 (The meandering streams SFT). The formal definition of the meandering streams shift $X_{\mathrm{MS}}$ uses an alphabet of 16 symbols as displayed in Figure 9. We will refer to symbol 1 as the blank symbol and to symbols 2 up to 16 as the non-blanks.

Now the 16 symbols can be placed next to each other only in a way that conserves the current indicated by the direction of the arrows. For example a symbol with an arrow pointing east, resp. west, at its right edge can only sit to the left of a symbol also having an arrow pointing east, resp. west, at its left edge, and a symbol with an arrow ending on its lower edge can sit above a symbol if and only if this has an arrow starting from its upper edge. 


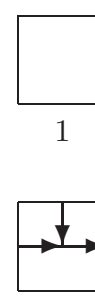

9
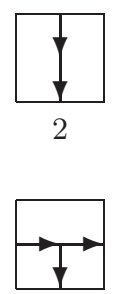

10
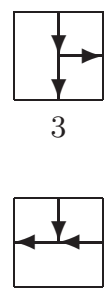

11
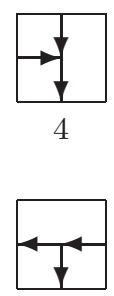

12

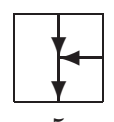

5

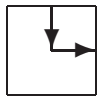

13

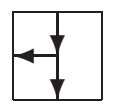

6

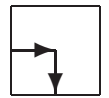

14
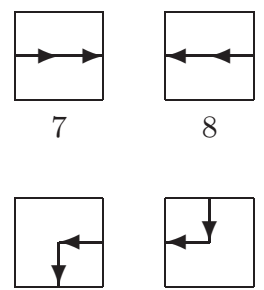

15

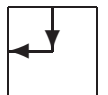

16

Figure 9. The alphabet of the meandering streams shift $X_{\mathrm{MS}}$.

Posing these nearest-neighbor restrictions as local rules, we obviously define a non-trivial $\mathbb{Z}^{2}$ SFT. Note that all allowed transitions are realized in some point, i.e. the corresponding transition matrices are already strictly essential. Next we prove the desired properties of our example.

Claim C.2. The meandering stream SFT $X_{\mathrm{MS}}$ is corner gluing at gap 2 in each of the 4 corners.

Proof. Since $X_{\mathrm{MS}}$ is a nearest-neighbor SFT and the horizontal, resp. vertical, transitions only depend on the arrows that are (are not) present at the vertical, resp. horizontal, borders of each symbol, we may prove the corner gluing property by filling the gap of width 2 between arbitrary configurations along the borders of the rectangle and the corner-shape region that appears in the definition of corner gluing.

In fact we will give a procedure to fill the gap between the following two infinite regions, forming a NW-corner in $\mathbb{Z}^{2}$ with parts of either two or three streams. Let $a, b \in \mathbb{Z}$, define an infinite corner-shape region $L^{\prime}:=\left\{\vec{u} \in \mathbb{Z}^{2} \mid u_{1}\left\langle a-2 \vee u_{2}\right\rangle\right.$ $b+2\}$ and an infinite rectangle $R^{\prime}:=\left\{\vec{v} \in \mathbb{Z}^{2} \mid v_{1} \geq a \wedge v_{2} \leq b\right\}$ and let $x, y \in X_{\mathrm{MS}}$ be two arbitrary points. Now we construct a new point $z \in X_{\mathrm{MS}}$ such that $\left.z\right|_{L^{\prime}}=\left.x\right|_{L^{\prime}}$ and $\left.z\right|_{R^{\prime}}=\left.y\right|_{R^{\prime}}$. The coordinates in $\mathbb{Z}^{2} \backslash\left(L^{\prime} \cup R^{\prime}\right)$ are filled with symbols as follows.

First, we put $\left.z\right|_{(a-1, b+1)}=15$, and for every $i \in \mathbb{N}$ we put $\left.z\right|_{(a-1, b+1-i)} \in\{2,3,5\}$ and $\left.z\right|_{(a-1+i, b+1)} \in\{8,12\}$. In this manner we have already nurtured all currents on the top-border of $R^{\prime}$ and nurtured/drained all currents on the left border of $R^{\prime}$. Second, we assign a pattern to the remaining coordinates, depending on the two symbols sitting to the left and above the coordinate $(a-2, b+2)$.

If $\left.z\right|_{(a-3, b+2)} \in\{1,2,4,6,14,16\}$, we use the first drawing of Figure 10, i.e. we put $\left.z\right|_{(a-2, b+2)} \in\{5,15\}$, and for all $i \in \mathbb{N}$ we put $\left.z\right|_{(a-2, b+2-i)} \in\{2,4,6\}$ and $\left.z\right|_{(a-2+i, b+2)} \in\{8,11\}$.

If $\left.z\right|_{(a-3, b+2)} \in\{3,7,9,10,13\}$, we use the second drawing of Figure 10, i.e. we put $\left.z\right|_{(a-2, b+2)} \in\{4,14\}$, and for all $i \in \mathbb{N}$ we put $\left.z\right|_{(a-2, b+2-i)} \in\{2,4,6\}$. Let $i^{\prime}:=\min \left\{i \in \mathbb{N}|z|_{(a-2+i, b+3)} \in\{2,3,4,5,6,10,12,14,15\}\right\}$. Then for every $i \in \mathbb{N}$ with $i<i^{\prime}$ put $\left.z\right|_{(a-2+i, b+2)}=1$, put $\left.z\right|_{\left(a-2+i^{\prime}, b+2\right)}=13$, and for $i>i^{\prime}$ put $\left.z\right|_{(a-2+i, b+2)} \in\{7,9\}$.

Similarily, if $\left.z\right|_{(a-3, b+2)} \in\{5,8,11,12,15\}$ and $\left.z\right|_{(a-2, b+3)} \in\{1,7,8,9,11,13,16\}$, we use the third drawing of Figure 10, and finally if $\left.z\right|_{(a-3, b+2)} \in\{5,8,11,12,15\}$ 


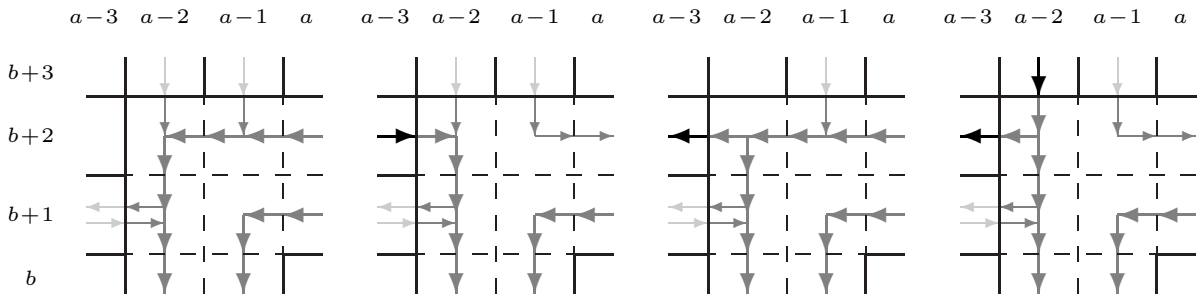

Figure 10. Corner gluing in the NW-corner.

and $\left.z\right|_{(a-2, b+3)} \in\{2,3,4,5,6,10,12,14,15\}$, we use the fourth drawing of Figure 10.

In all four cases we have constructed a new point $z \in X_{\mathrm{MS}}$ which combines the infinite patterns $\left.x\right|_{L^{\prime}}$ and $\left.y\right|_{R^{\prime}}$. Obviously this implies corner-gluing at gap size 2 for the NW-corner, as every finite NW-corner-gluing configuration (on finite regions $L$ and $R=[\vec{v}, \vec{w}])$ of gap size at least two can be reduced to the case treated above by choosing $a=v_{1}, b=w_{2}$ and extending $L$ to $L^{\prime} \supsetneq L$ and $R$ to $R^{\prime} \supsetneq R$ (keeping larger parts of the points $x, y$ ).

As the situation is completely symmetric in the east-west direction, one can flip the drawings in Figure 10 about their north-south axis to get appropriate fillings for the NE-corner. Similarily, because the situation is almost symmetric (except for the vertical current of a stream) in the north-south direction, one can produce corresponding fillings for the SW-corner, resp. SE-corner, by flipping the drawings in Figure 10, resp. those obtained for the NE-corner, about their east-west axis and reversing the direction of the current in all vertical parts afterwards.

Claim C.3. The meandering streams SFT $X_{\mathrm{MS}}$ does not have the UFP.

Proof. Note that $X_{\mathrm{MS}}$ contains the fixed point of all blanks. However, as a stream cannot stop at any coordinate in $\mathbb{Z}^{2}$, there are no points seeing non-blank symbols only at a finite non-empty subset of $\mathbb{Z}^{2}$. To show this, suppose there exists a point $x \in X_{\mathrm{MS}}$ and a non-empty finite subset $F \subsetneq \mathbb{Z}^{2}$ of coordinates such that $\left.x\right|_{\vec{v}} \neq 1$ for all $\vec{v} \in F$ but $\left.x\right|_{\vec{v}}=1$ for all $\vec{v} \in \mathbb{Z}^{2} \backslash F$. Let $b:=\max \{r \in \mathbb{Z} \mid \mathbb{Z} \times\{r\} \cap F \neq \emptyset\}$ be the top-most row in $\mathbb{Z}^{2}$ containing elements of $F$ and let $a:=\min \{c \in \mathbb{Z} \mid(c, b) \in F\}$ be the left-most coordinate in this row. Now the symbol at coordinate $(a, b)$ has to be a non-blank sitting beneath a blank and also sitting to the right of a blank symbol. Checking the alphabet of $X_{\mathrm{MS}}$ we find that $\left.x\right|_{(a, b)}=15$ - the only nonblank symbol with no arrow on its top nor its left border. Since by definition of $b$ all symbols in row $\mathbb{Z} \times\{b+1\}$ have to be blank, the only possibilities for $\left.x\right|_{(a+1, b)}$ are the symbols 8 or 12 . Continuing in this fashion we see that $\left.x\right|_{(a+i, b)} \in\{8,12\}$ for all $i \in \mathbb{N}$. Therefore $\left(a+\mathbb{N}_{0}\right) \times\{b\} \subseteq F$, which contradicts the finiteness of $F$.

This proves that in $X_{\mathrm{MS}}$ there is a unique way to fill in a rectangular frame of blanks, namely with all blanks. Thus it is not possible for any $g \in \mathbb{N}_{0}$ to glue together a non-blank symbol at the origin $U:=[\overrightarrow{0}, \overrightarrow{0}]=\{\overrightarrow{0}\}$ to a pattern of only blank symbols on $V:=\mathbb{Z}^{2} \backslash[-g \overrightarrow{\mathbb{1}}, g \overrightarrow{\mathbb{1}}]$, and thus $X_{\mathrm{MS}}$ does not have the UFP. 
Now we modify the meandering streams shift by splitting the blank symbol into $N \in \mathbb{N}$ distinct but completely interchangeable copies. This modified meandering streams SFT $X_{\mathrm{MS}}^{(N)}$ is still corner gluing at gap 2 in each of the 4 corners and still does not have the UFP. Moreover, for $N$ large enough it is not entropy minimal.

Claim C.4. For $N \geq 120$ the modified meandering streams SFT $X_{\mathrm{MS}}^{(N)}$ contains a proper subsystem of full entropy.

Proof. For $n \in \mathbb{N}$ we estimate the number of patterns in $\mathcal{L}_{[\overrightarrow{\mathbb{1}}, n \overrightarrow{\mathbb{1}}]}\left(X_{\mathrm{MS}}^{(N)}\right)$ containing a fixed number $0 \leq m \leq n^{2}$ of non-blank symbols. Those patterns are uniquely determined by specifying the finite parts of streams intersecting the solid block $B:=[\overrightarrow{\mathbb{1}}, n \overrightarrow{\mathbb{1}}]$ and putting in arbitrary blanks in the remaining positions. The key point in our argument is the fact that every non-blank in a point of $X_{\mathrm{MS}}^{(N)}$ is part of some infinite stream and that those have to hit the boundary of $B$ at least twice (once entering, once leaving).

This enables us to design an algorithm which allows a Turing machine to recreate every single pattern in $\mathcal{L}_{[\overrightarrow{\mathbb{1}}, n \overrightarrow{\mathbb{1}}]}\left(X_{\mathrm{MS}}^{(N)}\right)$ given a specific piece of input consisting of a finite list $L \subseteq B$ of coordinates, an $m$-tuple $\mathcal{I}$ of instructions taken from a set of 120 different commands and an $\left(n^{2}-m\right)$-tuple $\mathcal{N} \in\{1,2, \ldots, N\}^{n^{2}-m}$ of numbers specifying blank symbols. The list $L$ is a subset of the boundary $\partial B:=$ $B \backslash[2 \overrightarrow{\mathbb{1}},(n-1) \overrightarrow{\mathbb{1}}] \subsetneq \mathbb{Z}^{2}$ of $B$ that contains a starting coordinate for every connected finite part of a stream seen in $B$. Let $L$ be given an ordering by the lexicographic ordering on $\mathbb{Z}^{2}$. Every instruction in $\mathcal{I}$ is itself a 3 -tuple $\left(a_{i}, f_{i}, d_{i}\right)(1 \leq i \leq m)$, where $a_{i} \in\{2,3, \ldots, 16\}$ represents one of the 15 non-blanks in the alphabet of $X_{\mathrm{MS}}^{(N)}, f_{i} \in\{0,1\}$ is a flag that signals either "revert" or "continue," and $d_{i} \in$ $\{$ up, down, right, left $\}$ is one of the four standard directions in $\mathbb{Z}^{2}$. Now the Turing machine processes its input and puts down symbols on the solid block $B$ as follows: The machine starts by moving its writing-head to the coordinate given by the first entry in the list $L$ (if $L$ is empty, the machine skips the first part of the algorithm and continues with placing the blank symbols; see below). There it puts down the symbol $a_{1}$ from the first instruction in $\mathcal{I}$, starting a finite part of some stream. If $f_{1}$ is "continue" it moves its writing-head one step in the direction given by $d_{1}$, where it executes the next instruction in the same manner. If some $f_{i}$ is "revert," the machine moves back along the non-blank symbols written so far until it comes to the first junction - one of the symbols $\{3,4,5,6,9,10,11,12\}$ as in Figure 9 - where one of the three branches is a dead-end (i.e. the branch points to a place still inside $B$ but where the machine has not already placed another non-blank symbol). From there the machine moves one step in the direction specified by $d_{i}$ and continues with the $(i+1)$ th instruction. If there is no dead-end, the machine moves its writinghead to the next coordinate from the list $L$, where it starts another stream using the next instruction from $\mathcal{I}$. After executing all commands in $\mathcal{I}$, the machine has placed exactly $m$ non-blanks. It then jumps to coordinate $\overrightarrow{\mathbb{1}}$, from which it starts filling in the remaining $n^{2}-m$ coordinates of $B$ with blank symbols as specified by the numbers in $\mathcal{N}$.

As every pattern in $\mathcal{L}_{[\overrightarrow{1}, n \overrightarrow{\mathbb{1}}]}\left(X_{\mathrm{MS}}^{(N)}\right)$ can be created by our Turing machine using a particular input, the number of different "programs" gives an upper bound on the number of elements in $\mathcal{L}_{[\overrightarrow{1}, n \overrightarrow{1}]}\left(X_{\mathrm{MS}}^{(N)}\right)$ and thus on the entropy of $X_{\mathrm{MS}}^{(N)}$. Overestimating the number of different lists $L$ with the number of subsets of the boundary $\partial B$, 
summing over programs with exactly $m$ instructions $\left(0 \leq m \leq n^{2}\right)$, and assuming $N \geq 120$, we get

$$
\left|\mathcal{L}_{[\overrightarrow{\mathbb{1}}, n \overrightarrow{\mathbb{1}}]}\left(X_{\mathrm{MS}}^{(N)}\right)\right| \leq 2^{|\partial B|} \cdot \sum_{m=0}^{n^{2}}\left(120^{m} \cdot N^{\left(n^{2}-m\right)}\right) \leq 2^{4 n-4} \cdot\left(n^{2}+1\right) \cdot N^{n^{2}} .
$$

As a lower bound we take the number of different patterns containing only blanks, so $\left|\mathcal{L}_{[\overrightarrow{\mathbb{1}}, n \overrightarrow{\mathbb{1}}]}\left(X_{\mathrm{MS}}^{(N)}\right)\right| \geq N^{n^{2}}$. Putting both bounds together we see that $h\left(X_{\mathrm{MS}}^{(N)}\right)=\log N$ for all $N \geq 120$. As $X_{\mathrm{MS}}^{(N)}$ contains the full $\mathbb{Z}^{2}$ shift on $N$ (blank) symbols as a proper subshift, we are done.

\section{ACKNOWLEDGEMENTS}

The authors thank the anonymous referee for helpful comments and especially for pointing out an error in the original formulation of one of our propositions. The first author gratefully acknowledges the support and hospitality of the Centro de Modelamiento Matemático, Universidad de Chile, during this work.

\section{REFERENCES}

1. P. Balister, B. Bollobás and A. Quas, Entropy along convex shapes, random tilings and shifts of finite type, Illinois J. Math. 46 (2002), no. 3, 781-795. MR1951240 (2003j:37023)

2. A. Bertrand, Specification, synchronization, average length, in Coding theory and applications (Cachan, 1986), volume 311 of Lecture Notes in Comput., pages 86-95, Springer-Verlag, Berlin-New York, 1988. MR960710 (89i:94022)

3. M. Boyle, Lower entropy factors of sofic systems, Ergodic Theory Dynam. Systems 3 (1983), no. 4, 541-557. MR753922 (85m:54014)

4. M. Boyle and M. Schraudner, $\mathbb{Z}^{d}$ group shifts and Bernoulli factors, Ergodic Theory Dynam. Systems 28 (2008), no. 2, 367-387. MR2408383

5. M. Boyle and M. Schraudner, $\mathbb{Z}^{d}$ shifts of finite type without equal-entropy Bernoulli factors, J. Difference Equ. Appl. 15 (2009), no. 1, 47-52. MR2484416

6. R. Burton and J. Steif, Non-uniqueness of measures of maximal entropy for subshifts of finite type, Ergodic Theory Dynam. Systems 14 (1994), no. 2, 213-235. MR1279469 (95f:28023)

7. M. Denker, C. Grillenberger and K. Sigmund, Ergodic theory on compact spaces, Lecture Notes in Mathematics 527, Springer-Verlag, Berlin-New York, 1976. MR0457675 (56:15879)

8. A. Desai, Subsystem entropy for $\mathbb{Z}^{d}$ sofic shifts, Indagationes Mathematicae 17 (2006), no. 3, 353-360. MR2321105

9. A. Desai, A class of $\mathbb{Z}^{d}$ shifts of finite type which factor onto lower entropy full shifts, Proc. AMS, 137 (2009), no. 8, 2613-2621. MR2497473

10. E. Glasner, J.-P. Thouvenot and B. Weiss, Entropy theory without a past, Ergodic Theory Dynam. Systems 20 (2000), no. 5, 1355-1370. MR.1786718 (2001h:37011)

11. M. Hochman, On the dynamics and recursive properties of multidimensional symbolic systems, Invent. Math. 176 (2009), no. 1, 131-167. MR2485881(2009m:37023)

12. M. Hochman, On the automorphism groups of multidimensional SFTs, Ergodic Theory Dynam. Systems, to appear.

13. M. Hochman and T. Meyerovitch, A characterization of the entropies of multidimensional shifts of finite type, Annals of Math., to appear.

14. A. Johnson and K. Madden, Factoring higher-dimensional shifts of finite type onto the full shift, Ergodic Theory Dynam. Systems 25 (2005), no. 3, 811-822. MR2142947(2006c:37008)

15. W. Krieger, On the subsystems of topological Markov chains, Ergodic Theory Dynam. Systems 2 (1982), no. 1, 195-202. MR693975 (85b:28020)

16. S. Lightwood, Morphisms from non-periodic $\mathbb{Z}^{2}$-subshifts. I. Constructing embeddings from homomorphisms, Ergodic Theory Dynam. Systems 23 (2003), no. 2, 587-609. MR 1972240 (2004a:37022) 
17. S. Lightwood, Morphisms from non-periodic $\mathbb{Z}^{2}$-subshifts. II. Constructing homomorphisms to square-filling mixing shifts of finite type, Ergodic Theory Dynam. Systems 24 (2004), no. 4, 1227-1260. MR2085910(2005c:37021)

18. S. Lightwood and N. Ormes, Bounded orbit injections and suspension equivalence for minimal $\mathbb{Z}^{2}$ actions, Ergodic Theory Dynam. Systems 27 (2007), no. 1, 153-182. MR 2297092

19. D. Lind and B. Marcus, Introduction to symbolic dynamics and coding. Cambridge University Press, Cambridge, 1995. MR1369092 (97a:58050)

20. B. Marcus, Factors and extensions of full shifts, Monatsh. Math. 88 (1979), no. 3, 239-247. MR553733 (81g:28023)

21. B. Marcus, Sofic systems and encoding data, IEEE Trans. Inform. Theory 31 (1985), 366-377. MR794434(86m:94021)

22. N. Markley and M. Paul, Maximal measures and entropy for $\mathbb{Z}^{\nu}$ subshifts of finite type, Classical mechanics and dynamical systems, Lecture Notes in Pure and Appl. Math., 70 (1981), 135-157. MR640123 (83c:54059)

23. S. Mozes, Tilings, substitution systems and dynamical systems generated by them, J. Analyse Math. 53 (1989), 139-186. MR:1014984 (91h:58038)

24. A. Quas and A. Sahin, Entropy gaps and locally maximal entropy in $\mathbb{Z}^{d}$ subshifts, Ergodic Theory Dynam. Systems 23 (2003), 1227-1245. MR1997974(2004e:37027)

25. A. Quas and P. Trow, Subshifts of multi-dimensional shifts of finite type, Ergodic Theory Dynam. Systems 20 (2000), no. 3, 859-874. MR1764932(2001d:37011)

26. R.M. Robinson, Undecidability and non-periodicity of tilings of the plane, Inventiones Math. 12 (1971), 177-209. MR0297572(45:6626)

27. E.A. Robinson and A. Sahin, On the existence of Markov partitions for $\mathbb{Z}^{d}$ actions, J. London Math. Soc. (2) 69 (2004), no. 3, 693-706. MR2050041 (2005a:37028)

28. K. Schmidt, Algebraic $\mathbb{Z}^{d}$ actions, Lecture notes of PIMS Distinguished Chair Lectures, Pacific Institute for the Mathematical Sciences (2002), 61 pages.

29. K. Thomsen, On the structure of a sofic shift space, Trans. Amer. Math. Soc. 356 (2004), no. 9, 3557-3619. MR2055747|(2005a:37019)

30. T. Ward, Automorphisms of $\mathbb{Z}^{d}$-subshifts of finite type, Indag. Math., New Ser. 5 (1994), no. 4, 495-504. MR 1307966 (97a:28014)

Department of Mathematics, University of Maryland, College Park, Maryland $20742-4015$

E-mail address: mmb@math.umd.edu

$U R L$ : www. math. umd. edu/ mmb

Department of Mathematics, University of British Columbia, 1984 Mathematics Road, Vancouver, British Columbia, Canada V6T $1 \mathrm{Z} 2$

E-mail address: rpavlov@math.ubc.ca

$U R L$ : www.math.ubc.ca/ rpavlov/

Centro de Modelamiento Matematico, Universidad de Chile, Av. Blanco Encalada 2120, Piso 7, Santiago de Chile

E-mail address: mschraudner@dim.uchile.cl

URL: http://www.cmm.uchile.cl/ mschraudner/ 